\title{
Separated and Connected Maps
}

\author{
MARIA MANUEL CLEMENTINO ${ }^{1}$ and WALTER THOLEN ${ }^{2}$ \\ ${ }^{1}$ Departamento de Matemática, Apartado 3008, 3000 Coimbra, Portugal. \\ E-mail: mmc@mat.vc.pt \\ ${ }^{2}$ Department of Mathematics and Statistics, York University, Toronto, Canada M3J 1P3. \\ E-mail: tholen@mathstat.yorku.ca
}

(Received: 2 July 1996; accepted: 27 May 1997)

\begin{abstract}
Using on the one hand closure operators in the sense of Dikranjan and Giuli and on the other hand left- and right-constant subcategories in the sense of Herrlich, Preuß, Arhangel'skiǐ and Wiegandt, we apply two categorical concepts of connectedness and separation/disconnectedness to comma categories in order to introduce these notions for morphisms of a category and to study their factorization behaviour. While at the object level in categories with enough points the first approach exceeds the second considerably, as far as generality is concerned, the two approaches become quite distinct at the morphism level. In fact, left- and right-constant subcategories lead to a straight generalization of Collins' concordant and dissonant maps in the category $\mathcal{T}_{o p}$ of topological spaces. By contrast, closure operators are neither able to describe these types of maps in $\mathcal{T}_{o p}$, nor the more classical monotone and light maps of Eilenberg and Whyburn, although they give all sorts of interesting and closely related types of maps. As a by-product we obtain a negative solution to the ten-year-old problem whether the Giuli-Hušek Diagonal Theorem holds true in every decent category, and exhibit a counter-example in the category of topological spaces over the 1-sphere.
\end{abstract}

Mathematics Subject Classification (1991). 18B30, 54B30, 54D05, 54C10, 18A32, 18E40.

Key words: closure operator, left- and right-constant subcategory, separated and connected morphism, dissonant and concordant morphism.

\section{Introduction}

Ever since Eilenberg [16] and Whyburn [40] considered the factorization of a continuous map $f: X \rightarrow Y$ of compact Hausdorff spaces into a monotone quotient map (with connected fibres) followed by a light map (with hereditarily disconnected fibres), there have been studies on decomposition of maps into two maps whose fibres have opposite connectedness properties. Although Michael [30] established (monotone-quotient, light)-factorizations for $Y$ a $T_{1}$-space and $X$ any topological space, the two classes of maps do not give a factorization system in the category $\mathcal{T}_{o p}$ of topological spaces since the composite of monotone quotient maps may not be monotone. This defect is not present in Collins' [10] factorization of $f$ into a concordant quotient map (fibres are contained in components of the domain) followed by a dissonant map (fibres meet components of the domain in at most one point); see also $[15,11,34]$ for closely related factor- 
ization systems, and [20, 37, 4, 5] for (quite distinct) categorical generalizations thereof.

In this paper we follow a different direction, by first establishing a general notion of (dis)connectedness in an arbitrary category and then exploiting it in its slices, i.e. in the comma categories $\mathcal{X} / Y$ of objects over the fixed object $Y$, so that a 'connected' morphism $f: X \rightarrow Y$ in $\mathcal{X}$ is simply a 'connected' object in $\mathcal{X} / Y$. For the first part of this program, we offered two approaches in our recent paper [9] and compared them with each other:

(1) In the category $\mathcal{X}$ with a closure operator $c$, define $X$ to be $c$-connected (c-separated) if $\delta_{X}: X \rightarrow X \times X$ is $c$-dense ( $c$-closed) and obtain the corresponding subcategories $\nabla(c)$ and $\Delta(c)$ of $\mathcal{X}$.

(2) Follow the lead of Herrlich [20], Preuß [31] and Arhangels'kiǐ and Wiegandt [1] and define a Galois correspondence for full subcategories by

$$
\begin{aligned}
\mathcal{A} & \longmapsto r(\mathcal{A})=\{B \mid(\forall A \in \mathcal{A}) A \| B\} \\
l(\mathcal{B})=\{A \mid(\forall B \in \mathcal{B}) A \| B\} & \longleftrightarrow \mathcal{B},
\end{aligned}
$$

where $A \| B$ means that every morphism $A \rightarrow B$ is constant.

Under suitable conditions on $\mathcal{X}$, every left constant subcategory $l(\mathcal{B})$ is of the form $\nabla(c)$, and every right constant subcategory $r(\mathcal{A})$ is of the form $\Delta(c)$, for suitable closure operators $c$ (cf. [9], Theorems 7.6 and 7.7). In fact, approach (2) is very restrictive (in $\mathcal{T} o p$, for instance, the class of pathwise connected spaces fails to be left constant), while (1) is very general: the 'Diagonal Theorem' in $\mathcal{T}$ op [18] and its generalizations of [19,9] assert that every quotient-reflective subcategory is of the form $\Delta(c)$.

The suitable conditions on $\mathcal{X}$ alluded to above involve the existence of 'enough points'; for instance the assumption that the terminal object of $\mathcal{X}$ be a generator, which is far too restrictive in the slices of $\mathcal{X}$ (where 'points' of $f$ are sections of $f$ ). That is why, exploiting the notions (1), (2) for the comma categories $\mathcal{X} / Y$ and deriving a good 'fibrewise theory' from them is not a straightforward process. In fact, in the absence of enough points, the two approaches (1) and (2) become quite distinct and independent of each other, despite their many analogies and interconnections, as we show in this paper.

After a brief summary on the needed categorical tools in Section 1, we introduce the classes $\mathcal{C}$ onn $(c)$ and $\mathcal{S} e p(c)$ of $c$-connected and $c$-separated morphisms as the 'sliced' versions of $\nabla(c)$ and $\Delta(c)$ in Section 2 and discuss the question when they lead to a factorization system. Our summary result 3.10 gives two necessary and sufficient conditions, which are not very restrictive in categories of algebra but, not surprisingly, rarely found in topology. However, it is quite common to have $\mathcal{S e p}(c)$ being part of a factorization system, with the class $\mathcal{W C o n n}(c)$ of so-called weakly c-connected morphisms as its factorization com- 
panion, not the potentially smaller class of regular epimorphisms in $\mathcal{C}$ onn $(c)$ (cf. 3.5).

A similar effect occurs when looking at the 'sliced versions' $\operatorname{Conc}(\mathcal{A})$ and $\mathcal{D}$ iss $(\mathcal{A})$ of $l(r(\mathcal{A}))$ and $r(\mathcal{A})$, for a given subcategory $\mathcal{A}$ of $\mathcal{X}$ : the class of $\mathcal{A}$-dissonant morphisms is often part of a factorization system, but its factorization companion is not the class of $\mathcal{A}$-concordant regular epimorphisms but the potentially smaller class $\mathcal{S C o n c}(\mathcal{A})$ of strongly $\mathcal{A}$-concordant morphisms (see 4.4). Necessary and sufficient conditions for this system to allow for $(\operatorname{Conc}(\mathcal{A})$, $\mathcal{D} i s s(\mathcal{A})$ )-factorizations are given in 4.8 , where we also compare our approach with others mentioned earlier.

Relations between the classes $\operatorname{Conn}(c), \mathcal{S e p}(c)$ and $\operatorname{Conc}(\mathcal{A}), \mathcal{D} i s s(\mathcal{A})$ are discussed in Section 5. If $\Delta(c)=r(\nabla(c))$ has 'good' reflexion morphisms, $c$ separated morphisms are exactly the $\nabla(c)$-dissonant morphisms, and similarly for connected vis-a-vis concordant (see 5.6). However, in Section 6 we prove two strikingly negative results for closure operators: even for $\mathcal{A}=\{$ connected spaces $\}$ in $\mathcal{T}$ op, there is no closure operator $c$ such that the class of $\mathcal{A}$-dissonant maps ( $\mathcal{A}$-concordant quotient maps) is exactly the class of $c$-separated maps ( $c$ connected quotient maps, respectively); likewise, there is no closure operator which describes the light maps (monotone quotient maps) as the $c$-separated maps (c-connected quotient maps, respectively).

The first of the two main results of Section 6 leads to the solution of a problem which has been open since the appearance of [19] and was explicitly formulated in [14] (Problem 6.2): in a complete and cocomplete category $\mathcal{X}$ with a factorization system, is every strongly epireflective subcategory $\mathcal{B}$ of the form $\Delta(c)$ for some $c$ ? Taking for $\mathcal{X}$ the category $\mathcal{T} o p / S^{1}$ (with $S^{1}$ the 1 -sphere), we solve this problem negatively, taking in fact for $\mathcal{B}$ a right constant subcategory (cf. 7.1). The corresponding problem for left constant subcategories is also solved negatively by $\mathcal{T}_{o p} / S^{1}$ (cf. 7.2).

\section{Preliminaries on Factorization Systems and Closure Operators}

1.1. Let $\mathcal{M}$ be a class of morphisms in a category $\mathcal{X}$ which contains all isomorphisms and is closed under composition with isomorphisms. As in [14] we say that $\mathcal{X}$ has right $\mathcal{M}$-factorizations if every morphism $f$ of $\mathcal{X}$ factors as $f=m_{f} \cdot e_{f}$ with $m_{f} \in \mathcal{M}$ such that every commutative solid-arrow diagram

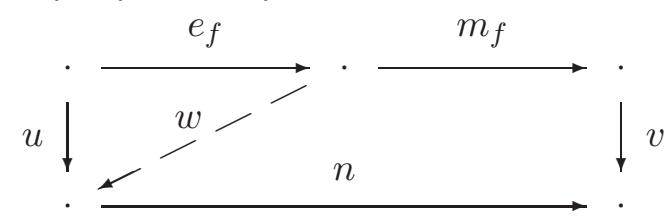

with $n \in \mathcal{M}$ admits a unique dotted fill-in morphism $w$ rendering both parts commutative. Obviously, the factorization of $f$ is unique up to isomorphism. Furthermore, it is easy to check that $\mathcal{M}$ must be closed under the formation of 
limits (in $\mathcal{X}^{2}$ ), in particular be stable under (multiple) pullback; however, $\mathcal{M}$ need not be closed under composition (see 1.4 below). The equivalence of the following statements was established in [35] and [29] (see also [28, 41]):

(i) $\mathcal{X}$ has right $\mathcal{M}$-factorizations;

(ii) $\mathcal{M}$, considered as a full subcategory of $\mathcal{X}^{2}$, is reflective;

(iii) (if $\mathcal{X}$ has pullbacks) $\mathcal{M}$ is stable under pullback, and for every object $Y \in \mathcal{X}$, the full subcategory $\mathcal{M} / Y$ of $\mathcal{X} / Y$ is reflective;

(iv) (if $\mathcal{X}$ has pullbacks) $\mathcal{M}$ is stable under pullback, and for every $f: X \rightarrow Y$ in $\mathcal{X}$, the inverse-image functor $f^{-1}(-): \mathcal{M} / Y \rightarrow \mathcal{M} / X$ has a left adjoint.

1.2. The left companion of $\mathcal{M}$ is the class $\mathcal{M}^{\perp}$ of all morphisms $e$ in $\mathcal{X}$ with $e \perp n$ for all $n \in \mathcal{M}$; here $e \perp n$ means that every solid-arrow commutative diagram

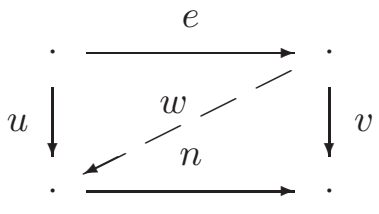

admits a unique diagonal $w$ as above (cf. [33, 17, 32]). It is well known that $\mathcal{M}^{\perp}$ is closed under the formation of colimits and under composition. Furthermore, if $\mathcal{X}$ has right $\mathcal{M}$-factorizations, then $\mathcal{M}^{\perp}=\left\{f \mid m_{f}\right.$ is an isomorphism $\}$ (cf. [14]).

1.3. In what follows the concepts dual to those of $1.1,1.2$ turn out to be equally important. Hence, for a given class $\mathcal{E}$ of morphisms containing the isomorphisms and being closed under composition with them, one says that $\mathcal{X}$ has left $\mathcal{E}$ factorizations if $\mathcal{X}^{o p}$ has right $\mathcal{E}$-factorizations; this means that every morphism $f$ factors as $f=m_{f} \cdot e_{f}$ with $e_{f} \in \mathcal{E}$ such that the diagonalization property depicted by

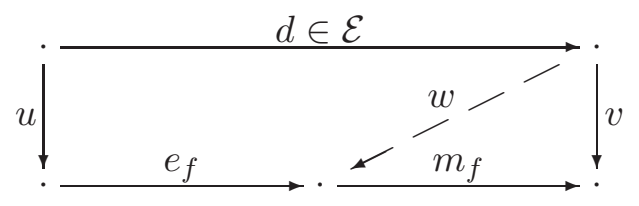

holds. The right companion of $\mathcal{E}$ is the class $\mathcal{E}_{\perp}$ of all morphisms $m$ with $e \perp m$ for all $e \in \mathcal{E}$, and one has $\mathcal{E}_{\perp}=\left\{f \mid e_{f}\right.$ is an isomorphism $\}$ in case $\mathcal{X}$ has left $\mathcal{E}$-factorizations.

1.4. The only reason for right $\mathcal{M}$ - or left $\mathcal{E}$-factorizations not to constitute an (orthogonal) factorization system for morphisms (as discussed in [17]) is the potential failure of $\mathcal{M}$ or $\mathcal{E}$ to be closed under composition. In fact, the 
following equivalent conditions for the given classes $\mathcal{E}, \mathcal{M}$ characterize $(\mathcal{E}, \mathcal{M})$ factorization systems:

(i) every $f$ factors as $f=m_{f} \cdot e_{f}$ with $e_{f} \in \mathcal{E}$ and $m_{f} \in \mathcal{M}$, and $e \perp m$ for all $e \in \mathcal{E}$ and $m \in \mathcal{M}$;

(ii) $\mathcal{X}$ has right $\mathcal{M}$-factorizations, and $\mathcal{M}$ is closed under composition;

(iii) $\mathcal{X}$ has left $\mathcal{E}$-factorizations, and $\mathcal{E}$ is closed under composition.

1.5. Throughout the rest of the paper, $\mathcal{X}$ is a finitely complete category with coequalizers of kernelpairs and a proper and stable $(\mathcal{E}, \mathcal{M})$-factorization system for morphisms; hence, in addition to the properties $1.4, \mathcal{M}$ is a class of monomorphisms of $\mathcal{X}$ and $\mathcal{E}$ is a class of epimorphisms of $\mathcal{X}$ stable under pullback. We note that the mono-assumption on $\mathcal{M}$ forces $\mathcal{E}$ to contain all regular epimorphisms; with the existence of kernelpairs and their coequalizers, this makes $\mathcal{X}$ to have (regular epi, mono)-factorizations, so that every strong epimorphism in $\mathcal{X}$ must be regular (cf. [25]). However, $\mathcal{X}$ need not be regular (cf. [2]), as regular epimorphisms are not assumed to be stable under pullback.

Since $(\mathcal{E}, \mathcal{M})$ is kept fixed, we refer to morphisms $m: M \rightarrow X$ in $\mathcal{M}$ as subobjects of $X$. With sub $X=\mathcal{M} / X$, every $f: X \rightarrow Y$ in $\mathcal{X}$ gives an image-preimage adjunction

$$
f(-) \dashv f^{-1}(-): \operatorname{sub} Y \longrightarrow \operatorname{sub} X .
$$

We use the usual lattice-theoretic notations in the preordered classes sub $X$.

1.6. (Cf. $[13,14]$.) A closure operator $c$ of $\mathcal{X}$ w.r.t. $(\mathcal{E}, \mathcal{M})$ is given by a family of functions $c_{X}: \operatorname{sub} X \rightarrow \operatorname{sub} X(X \in \mathcal{X})$ such that $m \leqslant c_{X}(m)$, $c_{X}(m) \leqslant c_{X}(n)$ if $m \leqslant n$, and $f\left(c_{X}(m)\right) \leqslant c_{Y}(f(m))$ for all $m, n \in \operatorname{sub} X$, $f: X \rightarrow Y$ in $\mathcal{X}$. For $m: M \rightarrow X$ in $\mathcal{M}$, we often write $c_{X}(m): c_{X}(M) \rightarrow X$, and we let $\frac{m}{c(m)}$ denote the $\mathcal{M}$-morphism $M \rightarrow c_{X}(M)$ with $c(m) \cdot \frac{m}{c(m)}=m$. As usual, $m$ is $c$-closed if $\frac{m}{c(m)}$ is an isomorphism, and $m$ is $c$-dense if $c(m)$ is an isomorphism; more generally, a morphism $f: X \rightarrow Y$ is $c$-dense if $f\left(1_{X}\right)$ is $c$-dense (in $Y$ ). The closure operator $c$ is idempotent if $c(m)$ is $c$-closed for all $m$, and it is weakly hereditary if $\frac{m}{c(m)}$ is $c$-dense for all $m$.

1.7. For any closure operator $c$ of $\mathcal{X}$ w.r.t. $(\mathcal{E}, \mathcal{M})$, the class $\mathcal{M}^{c}$ of $c$-closed subobjects is closed under limits (in $\mathcal{X}^{2}$ ), and the class $\mathcal{E}^{c}$ of $c$-dense morphisms is closed under colimits. With 1.4 one easily shows (cf. [14]):

(1) if $c$ is idempotent, then $\mathcal{X}$ has right $\mathcal{M}^{c}$-factorizations, hence $\mathcal{E}^{c}=\left(\mathcal{M}^{c}\right)^{\perp}$ is closed under composition;

(2) if $c$ is weakly hereditary, then $\mathcal{X}$ has left $\mathcal{E}^{c}$-factorizations, hence $\mathcal{M}^{c}=$ $\left(\mathcal{E}^{c}\right)_{\perp}$ is closed under composition; 
(3) if $c$ is idempotent (weakly hereditary) with $\mathcal{M}^{c}\left(\mathcal{E}^{c}\right)$ closed under composition, then $c$ is also weakly hereditary (idempotent, respectively).

1.8. For every closure operator $c$, a composite $d \cdot e$ belongs to $\mathcal{E}^{c}$ if $d \in \mathcal{E}$ and $e \in \mathcal{E}^{c}$, or if $d \in \mathcal{E}^{c}$ and $e \in \mathcal{E}$; conversely, $d \cdot e \in \mathcal{E}^{c}$ always implies $d \in \mathcal{E}^{c}$. The cancellation rule

(1) $d \cdot e \in \mathcal{E}^{c}, d \in \mathcal{M} \Rightarrow e \in \mathcal{E}^{c}$

holds if $c$ is hereditary, that is: if $c_{X}(m) \cong f^{-1}\left(c_{Y}(f \cdot m)\right)$ holds for all $m$ : $M \rightarrow X$ and $f: X \rightarrow Y$ in $\mathcal{M}$; in fact, $c$ is hereditary if and only if $c$ is weakly hereditary and (1) holds (cf. [14]). If $c_{X}\left(f^{-1}(n)\right) \cong f^{-1}\left(c_{Y}(n)\right)$ holds for all morphisms $f: X \rightarrow Y$ in $\mathcal{X}$ and $n \in \operatorname{sub} Y$, then $c$ is modal; equivalently, $c$ is hereditary and

(2) $\mathcal{E}^{c}$ is stable under pullback.

Consequently, an idempotent closure operator $c$ is modal if and only if $\left(\mathcal{E}^{c}, \mathcal{M}^{c}\right)$ is a stable factorization system of $\mathcal{X}$ (cf. [14]); such closure operators are called universal (cf. [3]).

1.9. Closedness of $\mathcal{E}^{c} \cap \mathcal{M}$ under (finite) products is easier to achieve; one just needs $c$ to be (finitely) productive, that is: $c_{X}\left(\prod_{i \in I} m_{i}\right) \cong \prod_{i \in I} c_{X_{i}}\left(m_{i}\right)$ for all $m_{i} \in \operatorname{sub} X_{i}$ with $X=\prod_{i \in I} X_{i}$ in $\mathcal{X}$ (and $I$ finite). In fact, for $c$ weakly hereditary, this is a necessary condition for $\mathcal{E}^{c} \cap \mathcal{M}$ to be closed under (finite) products (in $\mathcal{X}^{2}$ ).

Certainly, since $f \times f=(f \times 1)(1 \times f)$ is the composite of two pullbacks of $f$, for a universal closure operator $c$ the class $\mathcal{E}^{c}$ is closed under finite products. However, in categories where products have 'enough sections' (including $\mathcal{T}_{o p}$ ), every idempotent closure operator is finitely productive (cf. [14] 4.10).

1.10. For every $Z \in \mathcal{X}$, the comma category $\mathcal{X} / Z$ inherits any given factorization structure from $\mathcal{X}$. Specifically, for our (proper and stable) factorization system $(\mathcal{E}, \mathcal{M})$ of $\mathcal{X},\left(\mathcal{E}_{Z}, \mathcal{M}_{Z}\right)$ is a (proper and stable) factorization system of $\mathcal{X} / Z$, with $\mathcal{E}_{Z}$ and $\mathcal{M}_{Z}$ denoting the inverse images of $\mathcal{E}$ and $\mathcal{M}$, respectively, under the forgetful functor $\mathcal{X} / Z \rightarrow \mathcal{X}$. Furthermore, a closure operator $c$ of $\mathcal{X}$ w.r.t. $(\mathcal{E}, \mathcal{M})$ induces a closure operator $c^{Z}$ of $\mathcal{X} / Z$ w.r.t. $\left(\mathcal{E}_{Z}, \mathcal{M}_{Z}\right)$ : for every $m: g \rightarrow h$ in $\mathcal{M}_{Z}$ with $h: X \rightarrow Z$ in $\mathcal{X}$ (hence $g=h \cdot m$ ), $c_{h}(m): f \rightarrow h$ in $\mathcal{M}_{Z}$ has underlying $\mathcal{X}$-morphism $c_{X}(m)$ with $f=h \cdot c_{X}(m)$. Obviously, $c^{Z}$ is idempotent, (weakly) hereditary, or modal if the respective property holds for $c$.

\section{Separated and Connected Morphisms}

2.1. Let $c$ be a closure operator w.r.t. $(\mathcal{E}, \mathcal{M})$. An object $X$ of $\mathcal{X}$ is $c$-separated (or c-Hausdorff, cf. [8]) if $\delta_{X}=\left\langle 1_{X}, 1_{X}\right\rangle: X \rightarrow X \times X$ is $c$-closed. (Note that 
the split monomorphism $\delta_{X}$ belongs to $\mathcal{M}$ since $\mathcal{E}$ is a class of epimorphisms.) $X$ is c-connected (cf. [38,9]) if $\delta_{X}$ is $c$-dense. A morphism $f: X \rightarrow Y$ of $\mathcal{X}$ is c-separated (c-connected) if $f$ is $c^{Y}$-separated $\left(c^{Y}\right.$-connected, resp.) as an object of $\mathcal{X} / Y$ (cf. 1.10); equivalently, if the morphism $\delta_{f}=\left\langle 1_{X}, 1_{X}\right\rangle: X \rightarrow X \times_{Y} X$ is $c$-closed (c-dense, resp.). We put $r_{f}:=c\left(\delta_{f}\right): R_{f} \rightarrow X \times_{Y} X$ and $s_{f}:=$ $\frac{\delta_{f}}{r_{f}}: X \rightarrow R_{f}$, and we let $f_{1}, f_{2}: X \times_{Y} X \rightarrow X$ denote the projections of the kernelpair of $f$. One easily checks that the following conditions are equivalent (cf. [8]):

(i) $f$ is $c$-separated;

(ii) $f_{1} \cdot r_{f}=f_{2} \cdot r_{f}$;

(iii) for all $u, v: Z \rightarrow X$ and $m \in \operatorname{sub} Z$ with $u \cdot m=v \cdot m$ and $f \cdot u=f \cdot v$ one has $u \cdot c_{Z}(m)=v \cdot c_{Z}(m)$.

We note that the monomorphisms of $\mathcal{X}$, having trivial kernelpairs, are exactly the morphisms which are both $c$-separated and $c$-connected.

2.2. Since $\mathcal{M}^{c}$ is closed under limits (in $\mathcal{X}^{2}$ ), hence stable under (multiple) pullback, the class $\mathcal{S e p}(c)$ of all $c$-separated morphisms in $\mathcal{X}$ has the same properties (cf. [8]). Furthermore, for a composite morphism $h=(X \stackrel{f}{\longrightarrow} Y \stackrel{g}{\longrightarrow} Z)$ one has a commutative diagram

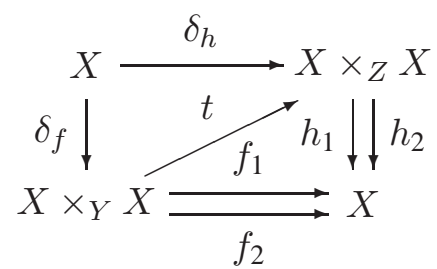

where the unique arrow $t$ with $h_{i} \cdot t=f_{i}$ is an equalizer of $f \cdot h_{1}, f \cdot h_{2}$. Hence there is a pullback diagram

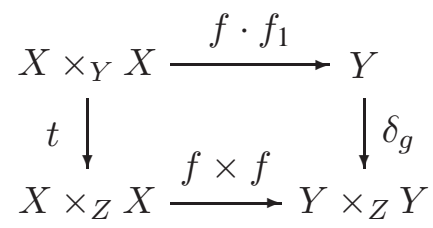

so that $t$ is $c$-closed when $g$ is $c$-separated. Composition-cancellation rules for $\mathcal{M}^{c}$ therefore give:

(1) $g \cdot f \in \mathcal{S} e p(c)$ implies $f \in \mathcal{S} e p(c)$;

(2) $f, g \in \mathcal{S} e p(c)$ implies $g \cdot f \in \mathcal{S} e p(c)$ if any of the two factors is monic or if $c$ is weakly hereditary.

(1) gives in particular that every morphism with $c$-separated domain is $c$-separated, since an object $X$ is $c$-separated iff $X \rightarrow 1$ with 1 terminal in $\mathcal{X}$ is $c$-separated. 
2.3. The class $\mathcal{C}$ onn $(c)$ of $c$-connected morphisms in $\mathcal{X}$ behaves less smoothly. Let $h=g \cdot f$ be as in 2.2, and in addition to diagrams (4), (5) consider

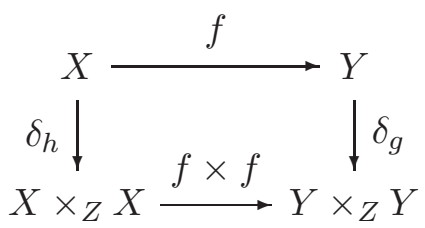

With $1.8,1.9$ one derives:

(1) $g \cdot f \in \mathcal{C}$ onn $(c)$ implies $f \in \mathcal{C}$ onn $(c)$ if $c$ is hereditary;

(2) $g \cdot f \in \mathcal{C}$ onn $(c)$ implies $g \in \mathcal{C}$ onn $(c)$ if $f \in \mathcal{E}$, or if $f \in \mathcal{E}^{c}$ and if $c$ is universal;

(3) $f, g \in \mathcal{C}$ onn $(c)$ implies $g \cdot f \in \mathcal{C}$ onn $(c)$ if $c$ is universal;

(4) $\mathcal{C}$ onn $(c)$ is closed under (finite) products in $\mathcal{X}^{2}$ if $c$ is (finitely) productive;

(5) for any pullback-stable class $\mathcal{K}$ of morphisms in $\mathcal{X}, \mathcal{C}$ onn $(c)$ is stable under pullback along $\mathcal{K}$-morphisms if $\mathcal{E}^{c}$ has this property.

Assertion (4) follows from $\delta_{f} \cong \prod_{i \in I} \delta_{f_{i}}$ for $f=\prod_{i} f_{i}$. For (5), one considers the diagram

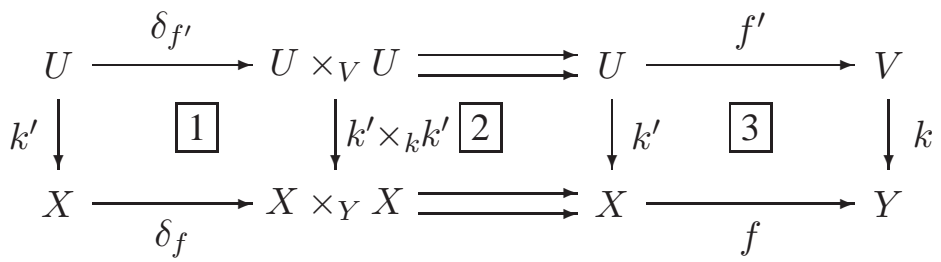

and notes that with 3 also 2 \& 3 and 1 are pullback diagrams.

2.4. Let $\Delta(c)$ be the full subcategory of $c$-separated objects in $\mathcal{X}$; it is closed under mono-sources in $\mathcal{X}$, hence regularly epireflective whenever $\mathcal{X}$ has products and is $\mathcal{E}$-cowellpowered (cf. [14], [9]). The (existing) $\Delta(c)$-reflexions decide whether the class

$$
\operatorname{Conn}^{*}(c):=\mathcal{C} \text { onn }(c) \cap \operatorname{Epi}^{*}(\mathcal{X})
$$

(with $\operatorname{Epi}^{*}(\mathcal{X})=\operatorname{Mono}(\mathcal{X})^{\perp}$ the class of strong, hence regular epimorphisms of $\mathcal{X})$ is the left companion of $\operatorname{Sep}(c)$ :

PROPOSITION. Let $c$ be hereditary in $\mathcal{X}$ and $\Delta(c)$ be reflective in $\mathcal{X}$. Then $\operatorname{Conn}^{*}(c)=\mathcal{S} e p(c)^{\perp}$ if and only if all $\Delta(c)$-reflexions are c-connected morphisms.

Proof. One always has $\mathcal{C o n n}^{*}(c) \subseteq \mathcal{S} e p(c)^{\perp}$ (as is shown more generally in 3.3 below). Furthermore, since $\operatorname{Mono}(\mathcal{X}) \subseteq \mathcal{S} e p(c)$, any $f: X \rightarrow Y$ in $\mathcal{S} e p(c)^{\perp}$ must belong to $\operatorname{Epi}^{*}(\mathcal{X})$. For the $\Delta(c)$-reflexion $e: X \rightarrow X^{\prime}$ of $X$, the morphism 
$X^{\prime} \rightarrow 1$ belongs to $\mathcal{S} \operatorname{ep}(c)$. Hence, the diagonalization property makes $e$ factor through $f$ :

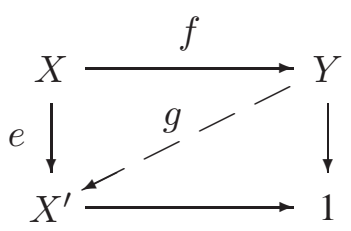

Assuming $e$ to belong to $\mathcal{C}$ onn $(c)$, we obtain $f \in \mathcal{C}$ onn $(c)$ with 2.3(1).

Conversely, having $\mathcal{C}_{o n n} *(c)=\mathcal{S} e p(c)^{\perp}$ we must show $e \in \mathcal{S} e p(c)^{\perp}$. But for the solid-arrow commutative diagram (2), $e$ factors through the pullback $\tilde{n}$ of $n$ along $v$ as

$$
e=\left(X \stackrel{t}{\longrightarrow} P \stackrel{\tilde{n}}{\longrightarrow} X^{\prime}\right) .
$$

As the composite of two $c$-separated morphisms, $P \rightarrow X^{\prime} \rightarrow 1$ is $c$-separated, hence $P \in \Delta(c)$. By the reflexion property, $t$ factors as $t=s \cdot e$. Hence the needed diagonal $w$ of (2) is obtained as the composite of $s$ with the pullback $\tilde{v}$ of $v$ along $n$.

COROLLARY 2.5. Let $c$ be hereditary and $\Delta(c)$ be reflective with c-connected reflexions. Then $\mathcal{C}_{\text {onn }}^{*}(c)$ is closed under composition and under the formation of colimits in $\mathcal{X}^{2}$. Furthermore, Conn ${ }^{*}(c)$ is stable under pullback along $\mathcal{M}$ morphisms if $\operatorname{Epi}^{*}(\mathcal{X})$ is, and for c (finitely) productive $\mathcal{C o n n}^{*}(c)$ is closed under (finite) products in $\mathcal{X}^{2}$ if $\operatorname{Epi}^{*}(\mathcal{X})$ is.

Connectedness of the $\Delta(c)$-reflexions comes by no means automatically. Even closedness under composition of $\mathcal{C o n n}^{*}(c)$ imposes a considerable restriction on $c$, as we shall show next.

\section{EXAMPLES 2.6.}

(1) In the category $\mathcal{T}$ op of topological spaces with its (surjective, embedding)factorization system, consider the usual (Kuratowski) closure $c=k$. A map $f: X \rightarrow Y$ is $k$-separated if and only if $X$ is fibrewise Hausdorff over $Y$ (cf. [23]), that is: if any pair of distinct points in a fibre of $f$ can be separated by disjoint open sets in $X$; and it is $k$-connected if and only if any two open sets in $X$ must meet if there is a fibre which meets each of the open sets. $\Delta(k)$ is the category of Hausdorff spaces. $\Delta(k)$-reflexions need not be $k$ connected, as is shown by the following space $X$. Its underlying set is $([0,1] \times \mathbb{N}) \cup\{\infty\}$, and a neighbourhood $U$ of $x$ in $X$ is required to contain for some $\delta>0$ the set

$-] a-\delta, a+\delta[\times\{n\}$ in case $x=(a, n)$ with $a \in] 0,1]$,

$-] 0, \delta[\times\{n, n+1\}$ in case $x=(0, n)$, 
- $] 0, \delta[\times\{n\}$ for infinitely many $n \in \mathbb{N}$ in case $x=\infty$.

Since every neighbourhood of $(0, n)$ meets every neighbourhood of $(0, n+$ $1)$, the Hausdorff reflexion $e: X \rightarrow X^{\prime}$ of $X$ satisfies $e(0, n)=e(0, n+1)$ for all $n \in \mathbb{N}$, hence $e(0, n)=y \in X^{\prime}$ is constant for all $n \in \mathbb{N}$. But also $e(\infty)=y$. In fact, for every neighbourhood $V$ of $y$ and every $\delta>0$ and $n \in$ $\mathbb{N}, e^{-1}(V) \cap(] 0, \delta[\times\{n\}) \neq \emptyset$, hence $e^{-1}(V)$ meets every neighbourhood of $\infty$; consequently, for every neighbourhood $W$ of $e(\infty), e^{-1}(W)$ meets $e^{-1}(V)$, hence $V \cap W \neq \emptyset$.

It is now easy to check that $X^{\prime}$ can be taken as the set (] $\left.\left.0,1\right] \times \mathbb{N}\right) \cup\{0\}$ provided with the quotient topology by $e: X \rightarrow X^{\prime}$ with $e(x)=x$ for $x \in] 0,1] \times \mathbb{N}$ and $e(x)=0$ otherwise. The $\left(k\right.$-)closure of $\delta_{e}$ in $X \times_{X^{\prime}} X$ is easily identified as the set

$$
\overline{\delta_{e}}=\delta_{e} \cup\{((0, n),(0, m))|| n-m \mid=1\} \neq X \times_{X^{\prime}} X
$$

so that $\delta_{e}$ fails to be $(k$-)dense.

(2) For $x \in X \in \mathcal{T}_{o p}$, let $\operatorname{comp}_{X}(x)$ denote the connected component of $x$ in $X$. For $M \subseteq X, \operatorname{comp}_{X}(M)=\bigcup_{x \in M} \operatorname{comp}_{X}(x)$ defines an idempotent and weakly hereditary but non-hereditary closure operator of $\mathcal{T}$ op. The $\nabla$ (comp) is the subcategory of connected spaces, and $\Delta$ (comp) is the subcategory of hereditarily disconnected spaces (i.e. $\operatorname{comp}_{X}(x)=\{x\}$ for all $x \in X$ ). A map $f: X \rightarrow Y$ is comp-connected (comp-separated) iff for all $x, y \in X$ with $f(x)=f(y), \operatorname{comp}_{Z}(x, y)$ meets the diagonal of $Z:=X \times_{Y} X$ $\operatorname{comp}_{Z}(x, y)$ meets the diagonal of $Z$ only if $x=y$, resp.); in terms of the reflector $C: \mathcal{T}_{o p} \rightarrow \Delta$ (comp), this means equivalently that the embedding $C \delta_{f}: C X \rightarrow C Z$ is a homeomorphism (that $p_{Z}^{-1}(C X)$ is $X$, when embedded into $Z$ via $\delta_{f}$; here $p_{Z}: Z \rightarrow C Z$ is the projection).

For a quotient map $f$ one has the implications

$$
f \text { monotone } \Rightarrow f \text { comp-connected } \Rightarrow f \text { concordant, }
$$

where $f$ concordant means equivalently that $C f$ is a homeomorphism. Neither of these two implications is reversible, not even if we restrict ourselves to the category of compact Hausdorff spaces: the map $f: I \rightarrow I$ with $f(x)=2 x$ for $x \leqslant \frac{1}{2}$ and $f(x)=2(1-x)$ for $x \geqslant \frac{1}{2}$ is a non-monotone (in fact: light) comp-connected quotient map, and the map $g: I \rightarrow I$ with $g(x)=\frac{1}{2}(3 x+1)$ for $x \leqslant \frac{1}{3}, g(x)=2-3 x$ for $\frac{1}{3} \leqslant x \leqslant \frac{2}{3}$ and $g(x)=\frac{1}{2}(3 x-2)$ for $x \geqslant \frac{2}{3}$ is a concordant (and light) quotient map, but not comp-connected. The map $g$ appears first in [24] where it was presented as the composite of two comp-connected maps. Hence, unlike $\mathcal{S} e p($ comp), the class $\mathcal{C}$ onn (comp) fails to be closed under composition. This also shows that $\mathcal{C}$ onn (comp) is not the orthogonal complement of $\mathcal{S e p}(\mathrm{comp})$; since the projections $p_{X}$ are comp-connected, we see in particular that the assumption of hereditariness is essential for 2.4. 
(3) It was shown in [9] that by taking for $c_{X}(M)$ the set of those points $x \in X$ for which there is a path in $X$ originating in $M \subseteq X$ and ending at $x$, one defines a weakly hereditary but non-hereditary closure operator $c$ of $\mathcal{T}_{o p}$ with $\nabla(c)$ the category of path-connected spaces and $\Delta(c)$ the category of hereditarily path-disconnected spaces. A map $f: X \rightarrow Y$ is $c$-connected iff for all $x, y \in X$ with $f(x)=f(y)$ there is a path $p: I \rightarrow X$ with

$$
\begin{aligned}
& p(0)=x, \quad p(1)=y, \\
& f(p(t))=f(p(1-t)) \text { for all } t \in[0,1] .
\end{aligned}
$$

The symmetry condition in $(\Lambda)$ is essential: mere existence of a path in $X$ connecting any two points of the same fibre of $f$ does not guarantee $c$-connectedness of $f$. In fact, the map $f: I \rightarrow S^{1}, \quad x \mapsto(\cos 2 \pi x, \sin 2 \pi x)$, winding the unit-interval around the 1 -sphere is not $c$-connected, since $I \times{ }_{S^{1}} I$ contains two isolated points outside the diagonal.

$f$ is $c$-separated iff for all $x, y \in X$ with $f(x)=f(y)$ a path $p$ with $(\wedge)$ exists only if $x=y$. $\mathcal{S} e p(c)$ is closed under composition (by 2.2(2)), but Conn $^{*}(c)$ is not: just consider the quotient maps $X \rightarrow S \rightarrow 1$ with $X$ the Topologist's Sine Curve and $S$ the Sierpinski space, both of which are $c$-connected while $X \rightarrow 1$ is not.

2.7. The true reason for failure of $\operatorname{Conn}^{*}(c)$ to be closed under composition in the previous example is explained more generally by:

PROPOSITION. For a closure operator c of $\mathcal{T}$ op (or of any category with suitable behaviour of points, $c f$. [9]), let Conn* ${ }^{*}(c)$ be closed under composition. Then $\nabla(c)$ is q-reversible, i.e., for every quotient map $f: X \rightarrow Y$ with $Y \in \nabla(c)$ and $f^{-1}(y) \in \nabla(c)$ for all $y \in Y$, also $X \in \nabla(c)$.

Proof. Since with $Y$ also $Y \rightarrow 1$ is $c$-connected, it suffices to prove that $f: X \rightarrow Y$ is $c$-connected, in order to conclude that then $(X \rightarrow Y \rightarrow 1)$ and therefore $X$ is $c$-connected. But the family of diagrams

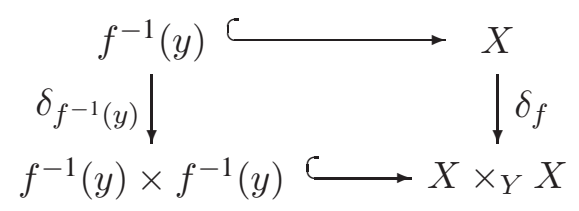

shows that $\delta_{f}$ is $c$-dense if all $\delta_{f^{-1}(y)}(y \in Y)$ are $c$-dense.

\section{The Connected-Separated Factorization}

3.1. From 2.2 one obtains with well-known Adjoint-Functor-Theorem methods the existence of right $\mathcal{S} \operatorname{ep}(c)$-factorizations. With 1.4 and 2.4 this gives: 
THEOREM. Let $\mathcal{X}$ be complete and $\mathcal{E}$-cowellpowered and $c$ be a closure operator w.r.t. $(\mathcal{E}, \mathcal{M})$. Then $\mathcal{X}$ has right $\mathcal{S} e p(c)$-factorizations, and these give an orthogonal factorization system if $c$ is weakly hereditary. For $c$ hereditary, these are $\left(\mathcal{C o n n}^{*}(c), \mathcal{S e p}(c)\right)$-factorizations if and only if every $\Delta(c)$-reflexion is $c$ connected.

Proof (sketch). For $f: X \rightarrow Y$, fix a representative system $\mathcal{E}_{X}$ of $\mathcal{E}$ morphisms with domain $X$ and form the intersection $t: T \rightarrow Y$ of all $s_{i}: S_{i} \rightarrow Y$ in $\mathcal{S} e p(c)$ for which there is $e_{i} \in \mathcal{E}_{X}$ with $f=s_{i} \cdot e_{i}$. Let $m \cdot p=g$ be an $(\mathcal{E}, \mathcal{M})$ factorization of the morphism $g: X \rightarrow T$ induced by the $e_{i}$; then $f=(t \cdot m) \cdot p$ is the desired right $\mathcal{S e p}(c)$-factorization of $f$.

3.2. Our next goal is to establish connected-separated factorizations more constructively, without resorting to Adjoint-Functor-Theorem methods. We assume $\mathcal{X}$ to have all coequalizers and, using the notation of 2.1, let $q_{f}: X \rightarrow Q_{f}$ be the coequalizer of $f_{1} \cdot r_{f}, f_{2} \cdot r_{f}$ and $h_{f}: Q_{f} \rightarrow Y$ the morphism with $h_{f} \cdot q_{f}=f$.

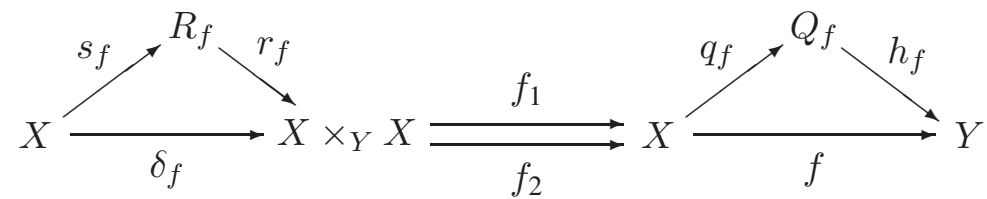

We observe that $q_{f}$ is an isomorphism iff $f_{1} \cdot r_{f}=f_{2} \cdot r_{f}$, that is: exactly if $f$ is $c$-separated. Let us call $f$ weakly c-connected if $h_{f}$ is an isomorphism, that is: if $f$ is a coequalizer of $f_{1} \cdot r_{f}, f_{2} \cdot r_{f}$. For the class $\mathcal{W C o n n}(c)$ of all weakly $c$-connected morphisms, one trivially has

$$
\operatorname{Conn}^{*}(c) \subseteq \mathcal{W C o n n}(c)
$$

for the reverse inclusion, see 3.6 below. [27]).

We first prove that the factorization $f=h_{f} \cdot q_{f}$ is functorial (in the sense of

LEMMA 3.3. For $v \cdot f=g \cdot u$ in $\mathcal{X}$ there is a uniquely determined morphism $w$ rendering the diagram

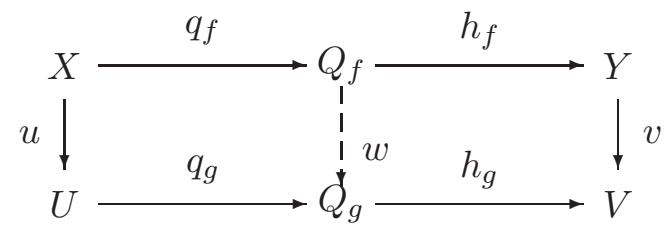

commutative. 
Proof. Since taking c-closures is functorial (cf. [14]), there is a unique morphism $z$ rendering the diagram

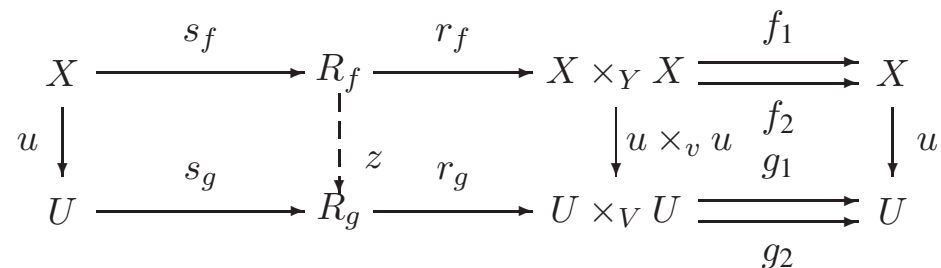

commutative. $w$ is induced by $z$.

LEMMA 3.4. If $c$ is weakly hereditary, $q_{f}$ is weakly c-connected for every $f$.

Proof. Let $q=q_{f}$ and $h=h_{f}$. Since $f=h \cdot q$, as in (4) one has an arrow $t$ producing the outer commutative diagram

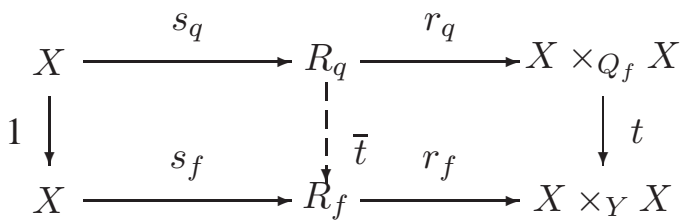

which then induces the fill-in arrow $\bar{t}$. Since $q \cdot f_{1} \cdot r_{f}=q \cdot f_{2} \cdot r_{f}$, there is also a canonical morphism $r: R_{f} \rightarrow X \times_{Q_{f}} X$ which satisfies $t \cdot r=r_{f}$ and $r \cdot s_{f}=\delta_{q}$. Since $c$ is weakly hereditary, so that $s_{f}$ is $c$-dense, the latter identity gives a morphism $s: R_{f} \rightarrow R_{q}$ with $s \cdot s_{f}=s_{q}$ and $r_{q} \cdot s=r$, and $s$ turns out to be inverse to $\bar{t}$. Consequently, $q$ being the coequalizer of $f_{1} \cdot r_{f}, f_{2} \cdot r_{f}$ is also the coequalizer of $q_{1} \cdot r_{q}, q_{2} \cdot r_{q}$.

THEOREM 3.5. Let $\mathcal{X}$ have coequalizers and $c$ be weakly hereditary. Then $\mathcal{X}$

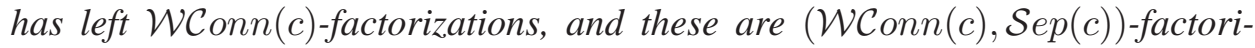
zations if and only if $\mathcal{W C o n n}(c)$ is closed under composition. In this case, $\Delta(c)-$ reflexions exist and belong to $\mathcal{W C}$ Conn $(c)$.

Proof. The first statement follows from 3.3 and 3.4, while for the second and third assertion one employs in addition 1.4 and 1.1, respectively, with the observation that $\mathcal{S e p}(c) / 1$ is isomorphic to $\Delta(c)$.

COROLLARY 3.6. Let $\mathcal{X}$ have coequalizers and $c$ be hereditary. Then the following statements are equivalent:

(i) $\mathcal{X}$ has $\left(\mathcal{C o n n}^{*}(c), \mathcal{S} e p(c)\right)$-factorizations;

(ii) $\Delta(c)$-reflexions exist and belong to $\mathcal{C}$ onn $n^{*}(c)$;

(iii) $\mathcal{W C o n n}(c)=\mathcal{C}_{\text {onn }} *(c)$ is closed under composition.

Proof. (i) $\Rightarrow$ (ii) follows from 1.1. (ii) $\Rightarrow$ (iii) From 3.2, 3.3 and 2.4 one has $\mathcal{W C o n n}(c) \subseteq \mathcal{S} e p(c)^{\perp}=\mathcal{C}$ onn $n^{*}(c) \subseteq \mathcal{W C}$ onn $(c)$,

with $\mathcal{S} e p(c)^{\perp}$ being closed under composition. (iii) $\Rightarrow$ (i) follows from 3.5 . 
EXAMPLES 3.7.

(1) The $b$-closure $b_{X}(M)$ of $M \subseteq X \in \mathcal{T}$ op contains those points $x \in X$ with $\overline{\{x\}} \cap M \cap U \neq \emptyset$ for every neighbourhood $U$ of $x$ in $X . b$ is a well-known hereditary closure operator of $\mathcal{T}_{o p}$ with $\Delta(b)$ the subcategory of $T_{0}$-spaces. Trivially, condition 3.6(ii) is satisfied, hence $\mathcal{T} o p$ has $\left(\mathcal{C} o n n^{*}(b), \mathcal{S} e p(b)\right)$ factorizations: every map $f: X \rightarrow Y$ factors through the quotient space given by $(x \sim y \Leftrightarrow f(x)=f(y) \& \overline{\{x\}}=\overline{\{y\}})$. Hence $f$ is $b$-separated iff this relation is discrete, and $f$ is $b$-connected iff $\sim$ coincides with the equivalence relation induced by $f$.

(2) Example 2.6(1) shows that condition (ii) of 3.6 does not hold in case $\mathcal{X}=$ $\mathcal{T} o p$ and $c=k$. Actually, in this case the (existing) $\Delta(c)$-reflexions may even fail to belong to $\mathcal{W C} \operatorname{conn}(c)$, so that the (existing) left $\mathcal{W C}$ onn $(c)$ factorizations fail to constitute a $(\mathcal{W C} \operatorname{onn}(c), \mathcal{S e p}(c))$-factorization system. In fact, in the notation of 2.6, the $\mathcal{W C}$ onn $(c)$-factor of the $\Delta(c)$-reflexion $e: X \rightarrow(] 0,1] \times \mathbb{N}) \cup\{0\}$ can be taken to be the quotient map $q=q_{e}$ : $X \longrightarrow(] 0,1] \times \mathbb{N}) \cup\{0, \infty\}$, with $q(x)=x$ for $x \in] 0,1] \times \mathbb{N}, q(x)=0$ for $x \in\{0\} \times \mathbb{N}$ and $q(\infty)=\infty$.

(3) For the closure operator $c=$ comp, the map $g$ of 2.6(2) was used in [24] to show that weakly $c$-connected maps need not be $c$-connected. Weakly $c$ connected maps are concordant quotient maps (see [24]), but not conversely: the map $f: I \rightarrow S^{1}$ of Example 2.6(3) is concordant and it is not weakly comp-connected.

(4) Also, for $c=p$ as in 2.6(3), $\mathcal{W C o n n}(c) \neq \mathcal{C o n n}^{*}(c)$. (For the space $X=$ $\{x, y, z, a, b\}$ with a subbase of open sets given by $\{x, a\},\{y, a, b\}\{z, b\}$, consider the quotient map identifying the points $x, y, z$.) Furthermore, not only $\mathcal{C o n n}^{*}(c)$ but also $\mathcal{W C}$ onn $(c)$ fails to be closed under composition (for the same reason as given in 2.6(3)).

(5) In general, closedness of $\mathcal{W C}$ onn $(c)$ under composition does not imply $\mathcal{W C o n n}(c)=\mathcal{C o n n}^{*}(c)$ :

In the category $\mathcal{S G} p h$ of spatial graphs (= sets with reflexive relations, maps preserve the relations) with its (surjective, embedding)-factorization structure, the up-closure

$$
\uparrow_{X} M=\{x \in X \mid(\exists y \in M) y \rightarrow x\}
$$

of $M \subseteq X$ defines a hereditary closure operator $\uparrow$ with $\Delta(\uparrow)$ the category of discrete graphs. A morphism $f: X \rightarrow Y$ is $\uparrow$-separated iff for all $x \leftarrow z \rightarrow y$ in $X$ with $f(x)=f(y)$ one has $x=y$, and it is $\uparrow$-connected iff for all $x, y \in X$ with $f(x)=f(y)$ there is $z \in X$ with $x \leftarrow z \rightarrow y$. Now, the (constant) $\Delta(\uparrow)$-reflexion of $X=\{\cdot \rightarrow \cdot \leftarrow \cdot\}$ is obviously not $\uparrow$-connected, hence $\left(\mathcal{C o n n}^{*}(\uparrow), \mathcal{S} \operatorname{ep}(\uparrow)\right)$-factorizations fail to exist in general. However, $\mathcal{S G}$ ph has $(\mathcal{W C}$ onn $(\uparrow), \mathcal{S} e p(\uparrow))$-factorizations which can be constructed as in 3.2: the equivalence relation $\sim$ generated by $R_{f}$ on $X$ is described by $x \sim y$ iff there is a finite zig-zag 


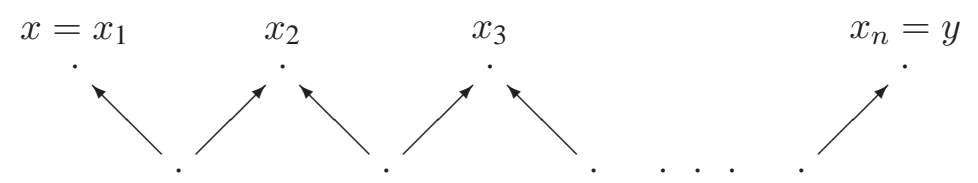

with all $x_{i}$ belonging to the same fibre of $f$; the quotient map $X \rightarrow X / \sim$ is weakly $\uparrow$-connected by definition, and the induced map $X / \sim \rightarrow Y$ is $\uparrow$-separated.

Failure of $r_{f}=c\left(\delta_{f}\right)$ to be an equivalence relation on $X$ in this example turns out to be the true reason for the inclusion $\mathcal{C}$ onn $n^{*}(c) \subset \mathcal{W} \mathcal{C}$ onn $(c)$ to be proper, as we show next.

3.8. Recall that $r \in \operatorname{sub}(X \times X)$ is an equivalence relation on $X$ if $r$ is reflexive $\left(\delta_{X} \leqslant r\right)$, symmetric $\left(r^{*} \leqslant r\right.$, with $r^{*}=\left\langle p_{2} \cdot r, p_{1} \cdot r\right\rangle$ and $p_{1}, p_{2}: X \times X \rightarrow X$ the projections), and transitive ( $r \circ r \leqslant r$, with $r \circ r$ given by the $\mathcal{M}$-part of an $(\mathcal{E}, \mathcal{M})$-factorization of $\left\langle p_{1} \cdot r \cdot \pi_{1}, p_{2} \cdot r \cdot \pi_{2}\right\rangle$ and $\pi_{1}, \pi_{2}$ the pullback projections of (14)).

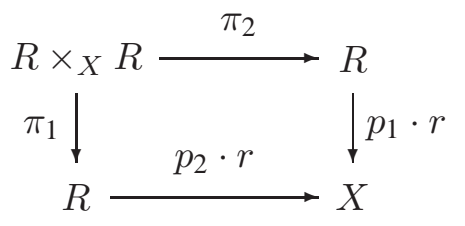

The equivalence relation $r$ is effective if $\left(p_{1} \cdot r, p_{2} \cdot r\right)$ is the kernelpair of some morphism.

In the context of 3.2 we say that $r_{f}$ is an effective equivalence relation on $X$ if $\left\langle f_{1} \cdot r_{f}, f_{2} \cdot r_{f}\right\rangle: R_{f} \rightarrow X \times X$ is one; this simply means that $\left(f_{1} \cdot r_{f}, f_{2} \cdot r_{f}\right)$ is the kernelpair of its coequalizer $q_{f}$.

PROPOSITION. If $\mathcal{X}$ has coequalizers and $c$ is weakly hereditary, the following statements are equivalent:

(i) $\mathcal{W C o n n}(c)=\mathcal{C o n n}^{*}(c)$;

(ii) for every morphism $f, r_{f}$ is an effective equivalence relation.

Proof. (i) $\Rightarrow$ (ii) We use the notation of the proof of Lemma 3.4 which, under condition (i), gives $q \in \mathcal{C}$ onn ${ }^{*}(c)$, so that $r_{q}$ and then $r=r_{q} \cdot s$ are isomorphisms. But the latter fact means that $\left(f_{1} \cdot r_{f}, f_{2} \cdot r_{f}\right) \cong\left(q_{1}, q_{2}\right)$ is a kernelpair, as desired.

(ii) $\Rightarrow$ (i) Every $f \in \mathcal{W C}$ onn $(c)$ is the coequalizer of $\left(f_{1} \cdot r_{f}, f_{2} \cdot r_{f}\right)$ which, under condition (ii), is the kernelpair of $f$, hence isomorphic to $\left(f_{1}, f_{2}\right)$. This is possible only if $r_{f}$ is an isomorphism, that is: if $f \in \mathcal{C o n n}^{*}(c)$.

3.9. The following proposition goes back to Barr [3] and has been proved as stated here in [14] 9.4: 
PROPOSITION. For a universal closure operator $c, r_{X}=c_{X \times X}\left(\delta_{X}\right)$ is an equivalence relation on $X$ for every $X \in \mathcal{X}$.

Since the proposition may be applied to the sliced category $\mathcal{X} / Y$, this shows that for a universal closure operator the equivalent conditions of Proposition 3.8 are satisfied, if equivalence relations in $\mathcal{X}$ are effective.

3.10. The following theorem follows with 3.6 and 3.8, and its corollary with 3.9:

THEOREM. Let $\mathcal{X}$ have coequalizers and let $c$ be hereditary. Then $\mathcal{X}$ has $\left(\mathcal{C o n n}^{*}(c), \mathcal{S e p}(c)\right)$-factorizations if and only if

(a) $\Delta(c)$-reflexions exist and are weakly c-connected, and

(b) for every morphism $f, r_{f}$ is an effective equivalence relation.

COROLLARY. Let $\mathcal{X}$ have coequalizers of equivalence relations, and let these be effective. Then, for a universal closure operator $c, \mathcal{X}$ has $\left(\mathcal{C o n n}^{*}(c), \mathcal{S}\right.$ ep $\left.(c)\right)$ factorizations.

EXAMPLE 3.11. For a unitary ring $R$, let $r$ be a preradical of $R$-modules. Hence, for every $X \in \mathcal{M o d}_{R}$, one has a submodule $r(X)$ such that $f(r(X)) \subseteq r(Y)$ for every $f: X \rightarrow Y$ in $\operatorname{Mod}_{R}$. Associated with $r$ is the maximal closure operator $\max ^{r}$, defined by $\max _{X}^{r}(M)=\pi^{-1}(r(X / M))$ for all $M \leqslant X$, with $\pi$ the projection onto $X / M$. The operator $\max ^{r}$ is idempotent iff $r$ is a radical (so that $r(X / r(X))=0$ for all $X$ ), and $\max ^{r}$ is weakly hereditary iff $r$ is idempotent (so that $r(r(X))=r(X)$ for all $X$ ); cf. [14]. Since $X \times_{Y} X / \Delta_{X} \cong \operatorname{ker} f$, the morphism $f: X \rightarrow Y$ is $\max ^{r}$-connected iff $\operatorname{ker} f$ is $r$-torsion (i.e., $r(\operatorname{ker} f)=$ $\operatorname{ker} f$ ), and it is $\max ^{r}$-separated iff $\operatorname{ker} f$ is $r$-torsionfree (i.e., $r(\operatorname{ker} f)=0$ ). Following the construction 3.2, one may factor $f$ as

$$
X \stackrel{q}{\longrightarrow} X / r(\operatorname{ker} f) \stackrel{h}{\longrightarrow} Y \text {. }
$$

If $r$ is idempotent, $q$ is $\max ^{r}$-connected, and if $r$ is a radical, $h$ is $\max ^{r}$-separated; in fact,

$$
r(\operatorname{ker} f)=r(\operatorname{ker} f / r(\operatorname{ker} f))=0 .
$$

The conditions on $r$ are in fact necessary: consider any $\left(\mathcal{C o n n}^{*}\left(\max ^{r}\right)\right.$, $\mathcal{S e p}\left(\max ^{r}\right)$ )-factorization of $X \rightarrow 0$, given by

$$
X \stackrel{q}{\longrightarrow} X / M \stackrel{h}{\longrightarrow} 0
$$

with $M \subseteq X$. Then $r(M)=M$, and $r(X) \subseteq M$ since the projection $q$ maps $r(X)$ into $r(X / M)=0$. Since trivially $r(M) \subseteq r(X)$, this shows $M=r(X)$, as desired. One therefore has: 
For a preradical $r, \mathcal{M o d}_{R}$ has $\left(\operatorname{Conn}^{*}\left(\max ^{r}\right), \mathcal{S e p}\left(\max ^{r}\right)\right)$-factorizations if and only if $r$ is an idempotent radical.

This condition on $r$ is equivalent to $\max ^{r}$ being idempotent and weakly hereditary. We note that if $\max ^{r}$ is hereditary, $\max ^{r}$ is actually already modal, and this is the case exactly if $r$ is hereditary (i.e., if $r(M)=r(X) \cap M$ for all $M \leqslant X \in \mathcal{M o d}_{R}$ ); cf. [14], 9.3.

\section{Dissonant and Concordant Morphisms}

4.1. (Cf. [9].) Recall that an object $P \in \mathcal{X}$ is preterminal if for all $X \in \mathcal{X}$ there is at most one morphism $X \rightarrow P$. A morphism $h$ with $(\mathcal{E}, \mathcal{M})$-factorization

$$
h=(X \stackrel{e}{\longrightarrow} P \stackrel{m}{\longrightarrow} Y)
$$

is constant if $P$ is preterminal. Writing $X \| Y$ iff every morphism $X \rightarrow Y$ is constant, one associates to full subcategories $\mathcal{A}, \mathcal{B}$ of $\mathcal{X}$ the right- and leftconstant subcategories

$$
r(\mathcal{A})=\{B|(\forall A \in \mathcal{A}) A| \mid B\}, \quad l(\mathcal{B})=\{A \mid(\forall B \in \mathcal{B}) A \| B\},
$$

respectively.

We now exploit these notions in the 'slices' of $\mathcal{X}$. A preterminal object in the comma category $\mathcal{X} / Z$ is simply a monomorphism in $\mathcal{X}$ with codomain $Z$. A morphism in $\mathcal{X} / Z$ given by the commutative triangle

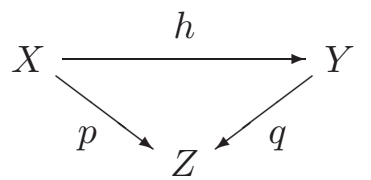

is constant in $\mathcal{X} / Z$ if $q \cdot m$ is monic in $\mathcal{X}$, with $m$ belonging to the $(\mathcal{E}, \mathcal{M})$-factorization of $f$. For a full subcategory $\mathcal{A}$ of $\mathcal{X}$, let $\mathcal{A} / Z$ denote the full subcategory of $\mathcal{X} / Z$ containing every $p: X \rightarrow Z$ with $X \in \mathcal{A}$. One can now form the full subcategories $r(\mathcal{A} / Z)$ and $l(r(\mathcal{A} / Z))$ of $\mathcal{X} / Z$. A morphism $q: Y \rightarrow Z$ in $\mathcal{X}$ is called $\mathcal{A}$-dissonant if $q \in r(\mathcal{A} / Z)$; equivalently, if for every morphism $h: X \rightarrow Y$ in $\mathcal{X}$ with $X \in \mathcal{A}$ and $h=m \cdot e$ its $(\mathcal{E}, \mathcal{M})$-factorization one has $q \cdot m$ monic. $p: X \rightarrow Z$ is called $\mathcal{A}$-concordant if $p \in l(r(\mathcal{A} / Z))$; equivalently, if in every factorization $p=q \cdot h$ with $q \mathcal{A}$-dissonant one has $q \cdot m$ monic (with $m$ as above). By $\operatorname{Diss}(\mathcal{A})$ and $\operatorname{Conc}(\mathcal{A})$ we denote the class of all $\mathcal{A}$-dissonant and of all $\mathcal{A}$-concordant morphisms, respectively, and we put

$$
\operatorname{Conc}^{*}(\mathcal{A}):=\operatorname{Conc}(\mathcal{A}) \cap \operatorname{Epi}^{*}(\mathcal{X})
$$

(cf. 2.4). With 1 denoting the terminal object, one has the following easily established object-morphism relations (see also 4.6(1) below):

LEMMA. For every $X \in \mathcal{X}$ : 
(1) $X \in r(\mathcal{A}) \Leftrightarrow(X \rightarrow 1) \in \operatorname{Diss}(\mathcal{A})$,

(2) $X \in l(r(\mathcal{A})) \Leftrightarrow(X \rightarrow 1) \in \operatorname{Conc}(\mathcal{A})$.

4.2. In what follows, we always consider a full subcategory $\mathcal{A}$ of $\mathcal{X}$ which is closed under $\mathcal{E}$-images, so that for $e: X \rightarrow Y$ in $\mathcal{E}$ and $X \in \mathcal{A}$ also $Y \in \mathcal{A}$. The class $\operatorname{Diss}(\mathcal{A})$ is easily seen to contain all monomorphisms of $\mathcal{X}$ and to be stable under pullback; furthermore, for $m$ monic and $q \in \mathcal{D} i s s(\mathcal{A})$, also $q \cdot m \in$ $\mathcal{D} i s s(\mathcal{A})$. These easily established properties are used to identify $\operatorname{Conc}^{*}(\mathcal{A})$ as the left companion of $\mathcal{D} i s s(\mathcal{A})$ :

PROPOSITION. $\operatorname{Conc}^{*}(\mathcal{A})=(\mathcal{D} \text { iss }(\mathcal{A}))^{\perp}$.

Proof. Given the solid-arrow diagram (2) with $e \in \mathcal{C}$ onc ${ }^{*}(\mathcal{A})$ and $n \in$ $\mathcal{D} i s s(\mathcal{A}), e$ factors through the pullback $n^{\prime}$ of $n$ along $v$ as $e=n^{\prime} \cdot t$. Since $n^{\prime} \in \mathcal{D} i s s(\mathcal{A}), n^{\prime} \cdot k$ is monic, where $k$ is the $\mathcal{M}$-part of $t$, and since $e \in \operatorname{Epi}^{*}(\mathcal{X})$, $n^{\prime} \cdot k$ must actually be an isomorphism. Hence $w=v^{\prime} \cdot\left(n^{\prime} \cdot k\right)^{-1}$ is the desired diagonal for (2). This shows $\mathcal{C}$ onc $c^{*}(\mathcal{A}) \subseteq \mathcal{D}$ iss $(\mathcal{A})^{\perp}$. For ' $\supseteq$ ', consider $p=q \cdot h$ with $p \in \operatorname{Diss}(\mathcal{A})^{\perp}$ and $q \in \operatorname{Diss}(\mathcal{A})$, and let $h=m \cdot e$ be an $(\mathcal{E}, \mathcal{M})$-factorization. Since $q \cdot m$ is $\mathcal{A}$-dissonant, there is $d$ with $q \cdot m \cdot d=1$ and $d \cdot p=e$ epic. Hence $d$ and $q \cdot m$ must be isomorphisms.

4.3. Our next goal is to establish $\left(\mathcal{C o n c}^{*}(\mathcal{A}), \mathcal{D} i s s(\mathcal{A})\right)$-factorizations. Let us assume that $\mathcal{X}$ has generalized coequalizers, i.e., simultaneous coequalizers of arbitrary families of parallel pairs of morphisms with common codomain. Given $f: X \rightarrow Y$, we may then form the coequalizer $p_{f}$ of all pairs $\left(a \cdot u_{1}, a \cdot u_{2}\right)$, where $a: A \rightarrow X$ is any morphism with domain in $\mathcal{A}$ and $\left(u_{1}, u_{2}\right)$ is the kernelpair of $u=f \cdot a$; then $f$ factors as $f=d_{f} \cdot p_{f}$ :

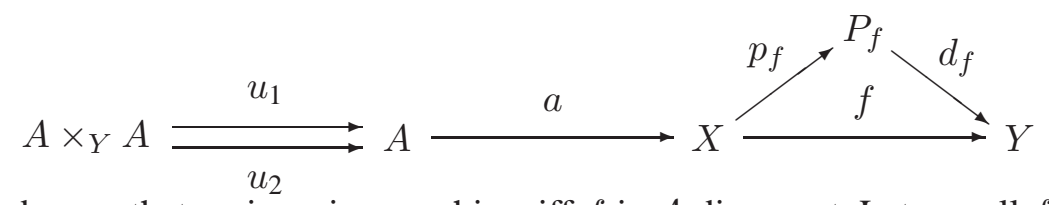

We observe that $p_{f}$ is an isomorphism iff $f$ is $\mathcal{A}$-dissonant. Let us call $f$ strongly $\mathcal{A}$-concordant if $d_{f}$ is an isomorphism. For the class $\mathcal{S C o n c}(\mathcal{A})$ of all strongly $\mathcal{A}$-concordant morphisms, one has:

LEMMA. $\operatorname{SConc}(\mathcal{A}) \subseteq \mathcal{C} \operatorname{Conc}^{*}(\mathcal{A})$.

Proof. Given $p=q \cdot h$ with $p \in \mathcal{S C o n c}(\mathcal{A})$ and $q \in \mathcal{D}$ iss $(\mathcal{A})$, it suffices to show $e \cdot a \cdot u_{1}=e \cdot a \cdot u_{2}$ for $h=m \cdot e$ with $e \in \mathcal{E}, m \in \mathcal{M}$ and $a, u_{i}$ as above. With $h \cdot a=m^{\prime} \cdot e^{\prime}$ an $(\mathcal{E}, \mathcal{M})$-factorization, one has $q \cdot m^{\prime}$ monic, hence $e^{\prime} \cdot u_{1}=e^{\prime} \cdot u_{2}$ and then $h \cdot a \cdot u_{1}=h \cdot a \cdot u_{2}$. But since $m$ is monic, this implies the desired equation, and one concludes the proof similarly to 4.2.

THEOREM 4.4. Let $\mathcal{X}$ have generalized coequalizers, and let the full subcategory $\mathcal{A}$ be closed under $\mathcal{E}$-images. Then $\mathcal{X}$ has left $\mathcal{S C o n c}(\mathcal{A})$-factorizations, 
and these are $(\mathcal{S C o n c}(\mathcal{A}), \operatorname{Diss}(\mathcal{A}))$-factorizations if and only if $\operatorname{SConc}(\mathcal{A})=$ $\operatorname{Conc}^{*}(\mathcal{A})$.

Proof. Given $f: X \rightarrow Y$ as in 4.3 , for every $A \rightarrow X$ with $A \in \mathcal{A}$ one has $A \times_{Y} A \cong A \times_{P_{f}} A$, which shows $p_{f} \in \mathcal{S C}$ onc $(\mathcal{A})$. Furthermore, as in 3.3, one easily shows that the factorization $f=d_{f} \cdot p_{f}$ is functorial. Consequently, one has left $\mathcal{S C o n c}(\mathcal{A})$-factorizations, and these form an orthogonal factorization system if and only if $\mathcal{S C o n c}(\mathcal{A})$ is closed under composition; in this case $\mathcal{S C o n c}(\mathcal{A})_{\perp}=$ $\operatorname{Diss}(\mathcal{A})$ by 1.3 and $\mathcal{S C o n c}(\mathcal{A})=\operatorname{Diss}(\mathcal{A})^{\perp}=\mathcal{C}_{\text {onc }}^{*}(\mathcal{A})$ by 4.2. Conversely, if the latter equations hold, $\mathcal{S C o n c}(\mathcal{A})$ must be closed under composition since $\operatorname{Diss}(\mathcal{A})^{\perp}$ has this property.

We note that the inclusion $\mathcal{S C O n c}(\mathcal{A}) \subseteq \mathcal{C} \operatorname{onc}^{*}(\mathcal{A})$ may be proper (see 4.9(2)).

In what follows we try to provide sufficient conditions for the existence of $(\operatorname{Conc}(\mathcal{A}), \operatorname{Diss}(\mathcal{A}))$-factorizations as well as handy descriptions for these classes of morphisms.

4.5. We call $f: X \rightarrow Y \mathcal{A}$-concordant in the sense of Collins [10] if for every $a: A \rightarrow Y$ in $\mathcal{M}$ with $A \in \mathcal{A}$, the pullback $f^{-1}(a): f^{-1}(A) \rightarrow X$ factors through an object of $\mathcal{A}$. This defines the class of morphisms $\mathcal{C} \mathcal{C}$ onc $(\mathcal{A})$, and we put $\mathcal{C} \operatorname{Conc}^{*}(\mathcal{A})=\mathcal{C} \mathcal{C o n c}(\mathcal{A}) \cap \operatorname{Epi}^{*}(\mathcal{X})$. Recall that $\mathcal{A}$ is a generating class of $\mathcal{X}$ if for every $X \in \mathcal{X}$ the family of all morphisms $A \rightarrow X$ with $A \in \mathcal{A}$ is jointly epic in $\mathcal{X}$.

LEMMA. If $\mathcal{A}$ is generating, then $\mathcal{C C O n c}{ }^{*}(\mathcal{A}) \subseteq \mathcal{S C o n c}(\mathcal{A})$.

Proof. Since $f \in \mathcal{C C O n c}^{*}(\mathcal{A})$ is a regular epimorphism and $\mathcal{A}$ is generating, it suffices to show that $f \cdot u=f \cdot v$ with $u, v: A \rightarrow X$ and $A \in \mathcal{A}$ implies $p_{f} \cdot u=p_{f} \cdot v$. Let $a^{\prime}: A^{\prime} \rightarrow Y$ be the $\mathcal{E}$-image of $f \cdot u=f \cdot v$; then $u$ and $v$ factor through the pullback $f^{-1}\left(a^{\prime}\right)$ which in turn factors through a morphism $\tilde{A} \rightarrow X$ with $\tilde{A} \in \mathcal{A}$. Consequently, $u$ and $v$ factor through the kernelpair $\tilde{A} \times_{Y} \tilde{A}$, which shows $p_{f} \cdot u=p_{f} \cdot v$.

4.6. Recall that $r(\mathcal{A})$ is always closed under monosources in $\mathcal{X}$ (cf. [9]); hence it is strongly epireflective if $\mathcal{X}$ has products and is $\mathcal{E}$-cowellpowered. It is therefore not very restrictive to assume that every $X \in \mathcal{X}$ has an $r(\mathcal{A})$-reflexion $\varrho_{X}: X \rightarrow$ $R X$.

\section{PROPOSITION.}

(1) A morphism $q: Y \rightarrow Z$ with $Y \in r(\mathcal{A})$ is $\mathcal{A}$-dissonant. Conversely, if $q$ is $\mathcal{A}$-dissonant and $Z \in r(\mathcal{A})$, then also $Y \in r(\mathcal{A})$.

(2) Every $r(\mathcal{A})$-reflexion is $\mathcal{A}$-concordant.

Proof. (1) Every morphism $a: A \rightarrow Y$ with $A \in \mathcal{A}$ and $Y \in r(\mathcal{A})$ is constant. If, without loss of generality, we assume $a \in \mathcal{M}, A$ must therefore be 
preterminal, and $q \cdot a$ is monic. Conversely, if $q \in \mathcal{D} i s s(\mathcal{A})$ and $Z \in r(\mathcal{A})$, for every $a: A \rightarrow Y$ with $A \in \mathcal{A}$ we have $q \cdot a$ monic and constant, so that $A$ is preterminal and $a$ is constant.

(2) If $\varrho_{X}=q \cdot h$ with $q \mathcal{A}$-dissonant and $h=m \cdot e$ an $(\mathcal{E}, \mathcal{M})$-factorization, one has $q \cdot m: B \rightarrow R X \mathcal{A}$-dissonant. Hence $B \in r(\mathcal{A})$ by (1), and the reflection property gives a morphism $d$ with $q \cdot m \cdot d=1$ and $d \cdot p=e$, which gives $q \cdot m$ iso (as in 4.2).

4.7. Let $r(\mathcal{A})$ be reflective in $\mathcal{X}$. A morphism $f: X \rightarrow Y$ is called $\mathcal{A}$ concentrated or $\mathcal{A}$-concordant in the sense of Herrlich, Salicrup and Vázquez [21] if the $r(\mathcal{A})$-reflexion $\varrho_{X}: X \rightarrow R X$ factors through $f$ as $\varrho_{X}=g \cdot f$ with $R f \cdot g=\varrho_{Y}$ (cf. [35], 4.1). This defines the class of morphisms $\mathcal{H C o n c}(\mathcal{A})$, and we put $\mathcal{H C} \operatorname{Conc}^{*}(\mathcal{A})=\mathcal{H C} \operatorname{Conc}(\mathcal{A}) \cap \operatorname{Epi}^{*}(\mathcal{X})$. Note that $\mathcal{H C}$ onc $(\mathcal{A})$ depends only on $r(\mathcal{A})$, not on $\mathcal{A}$; hence $\mathcal{H C o n c}(\mathcal{A})=\mathcal{H C} \operatorname{onc}(\operatorname{lr}(\mathcal{A}))$.

\section{PROPOSITION.}

(1) $\operatorname{Conc}^{*}(\mathcal{A}) \subseteq \mathcal{H C O n c}(\mathcal{A})$.

(2) $\mathcal{H C o n c}^{*}(\mathcal{A}) \subseteq \mathcal{C C O n c}^{*}(\mathcal{A})$ holds if and only if all $r(\mathcal{A})$-reflexions lie in $\operatorname{CC}$ onc $(\mathcal{A})$.

(3) If $\mathcal{A}$ contains all preterminal objects and if $r(\mathcal{A})$-reflexions lie in $\mathcal{C C}$ onc $(\mathcal{A})$, then $\mathcal{A}=l(r(\mathcal{A}))$ is left constant.

Proof. (1) For $f \in \mathcal{C o n c}^{*}(\mathcal{A})$ and $\varrho_{X}$ the $r(\mathcal{A})$-reflexion of $X$ one obtains with 4.1 and 4.2 the commutative diagram

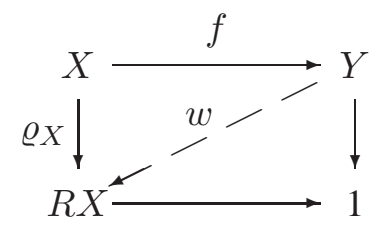

(2) By (1) and 4.6(2), the condition $\varrho_{X} \in \mathcal{C C} \operatorname{Conc}(\mathcal{A})$ for all $X$ is certainly necessary for $\mathcal{H C} \operatorname{onc}^{*}(\mathcal{A}) \subseteq \mathcal{C C} \operatorname{onc}(\mathcal{A})$. For its sufficiency, assume $w \cdot f=\varrho_{X}$, and consider $a: A \rightarrow Y$ in $\mathcal{M}$ with $A \in \mathcal{A}$. Since $f^{-1}(a) \leqslant \varrho_{X}^{-1}(w(a))$, the morphism $f^{-1}(a)$ factors through an object of $\mathcal{A}$ whenever $\varrho_{X}^{-1}(w(a))$ has this property.

(3) For $X \in l(r(\mathcal{A}))$, the $\mathcal{E}$-morphism $\varrho_{X}: X \rightarrow R X$ is constant, hence $R X$ is preterminal. Consequently, by hypothesis, $R X \in \mathcal{A}$, and $1_{X} \cong \varrho_{X}^{-1}\left(1_{R X}\right)$ factors through an $\mathcal{A}$-object, which forces $X$ to lie in $\mathcal{A}$.

THEOREM 4.8. Let $\mathcal{X}$ have generalized coequalizers, and let $\mathcal{A}$ be closed under $\mathcal{E}$-images and $r(\mathcal{A})$ reflective in $\mathcal{X}$. Then $\mathcal{X}$ has $(\mathcal{S C o n c}(\mathcal{A}), \mathcal{D}$ iss $(\mathcal{A})$ )-factorizations with

$$
\operatorname{SConc}(\mathcal{A})=\operatorname{Conc}^{*}(\mathcal{A}) \subseteq \mathcal{H C} \operatorname{Conc}^{*}(\mathcal{A}) \subseteq \mathcal{C C} \operatorname{Conc}^{*}(\mathcal{A})
$$


if and only if all $r(\mathcal{A})$-reflexions lie in $\mathcal{C C o n c}(\mathcal{A})$. In this case, for $\mathcal{A}$ generating in $\mathcal{X}$, all four morphism classes of $(*)$ coincide.

Proof. With 4.4 and 4.7, in the factorization $f=d_{f} \cdot p_{f}$ of 4.3 one has $p_{f} \in$

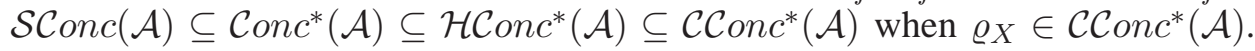
In order to show $d_{f} \in \mathcal{D} i s s(\mathcal{A})$, consider $a: A \rightarrow P_{f}$ in $\mathcal{M}$ with $A \in \mathcal{A}$. Since $p_{f} \in \mathcal{C C}$ onc $^{*}(\mathcal{A})$, the pullback $b=f^{-1}(a): B \rightarrow X$ factors through an $\mathcal{A}$-object. From the construction of $p_{f}$ one has $p_{f} \cdot b \cdot v_{1}=p_{f} \cdot b \cdot v_{2}$, with $v=f \cdot b$ and $\left(v_{1}, v_{2}\right)$ its kernelpair.

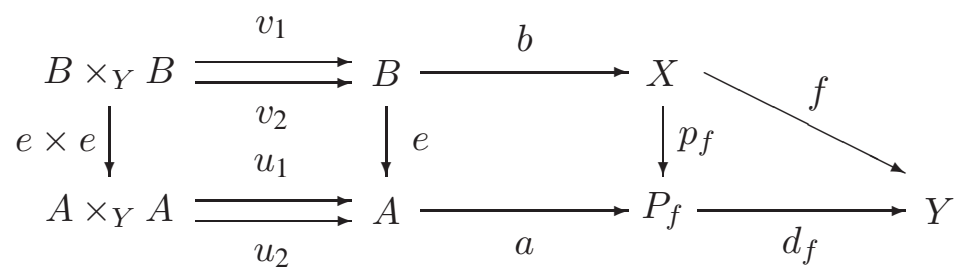

Hence $a \cdot u_{1} \cdot(e \times e)=a \cdot u_{2} \cdot(e \times e)$, with $\left(u_{1}, u_{2}\right)$ the kernelpair of $u:=d_{f} \cdot a$ and $e$ the pullback of $p_{f}$. Since $(e \times e)=(e \times 1)(1 \times e)$ is epic and $a$ is monic, this shows $u_{1}=u_{2}$, whence $d_{f} \cdot a$ is monic.

Since $r(\mathcal{A})$-reflexions lie in $\operatorname{Conc}^{*}(\mathcal{A})$, the necessity of the condition $\varrho_{X} \in$ $\mathcal{C C} \operatorname{onc}^{*}(\mathcal{A})$ for all $X$ is trivial. Furthermore, equality of the classes in $(*)$ in case $\mathcal{A}$ is generating follows with 4.5 .

\section{EXAMPLES 4.9.}

(1) For $\mathcal{X}=\mathcal{T}$ op and $\mathcal{A}=$ connected spaces $\}$, Theorem 4.8 is fully applicable; in particular, the four classes of $(*)$ coincide and describe the concordant quotient maps as defined in the Introduction. (Note that Collins [10] uses quasi-components instead of components when defining concordant maps.)

(2) Consider $\mathcal{X}=\mathcal{T}$ op and $\mathcal{A}=$ \{path-connected spaces $\}$; with $X$ the Topologist's Sine Curve, one has $f=(X \rightarrow 1) \in \mathcal{C}_{\text {onc }}^{*}(\mathcal{A})$ since $X \in \operatorname{lr}(\mathcal{A})=$ \{connected spaces\}, but $f \notin \mathcal{S C}$ onc $(\mathcal{A})$ since $P_{f}$ is the Sierpinski space. Hence, by Theorem 4.4, $\mathcal{T}_{\text {op }}$ does not have $\left(\operatorname{Conc}^{*}(\mathcal{A}), \mathcal{D}\right.$ iss $\left.(\mathcal{A})\right)$-factorizations.

(3) In the category $\mathcal{S G} p h$, let $\mathcal{A}=\nabla(\uparrow)$ be the subcategory of graphs $X$ such that for all $x, y \in X$ there is $z \in X$ with $x \leftarrow z \rightarrow y$ (cf. 3.7(5)). $\mathcal{A}$ fails to be left-constant, since $r(\mathcal{A})$ is the subcategory of discrete graphs and $l(r(\mathcal{A}))$ is the subcategory of all graphs in which any two points can be connected by an undirected path. $\operatorname{Diss}(\mathcal{A})$ coincides with the class $\mathcal{S} e p(\uparrow)$ of 3.7(5), and $\mathcal{C}_{\text {onc }}^{*}(\mathcal{A})=\mathcal{W} \mathcal{C}$ onn $(\uparrow)$. Hence $\mathcal{S G}$ ph has $(\mathcal{S C o n c}(\mathcal{A}), \mathcal{D}$ iss $(\mathcal{A}))$-factorizations with $\mathcal{S C o n c}(\mathcal{A})=\mathcal{C o n c}^{*}(\mathcal{A})$. But $r(\mathcal{A})$-reflexions may fail to be in $\operatorname{CC} \operatorname{conc}(\mathcal{A})$ (for the same reason as $\Delta(\uparrow)$-reflexions may fail to be $\uparrow$ connected); consequently, the inclusion $\mathcal{C} \mathcal{C o n c}^{*}(\mathcal{A}) \subset \mathcal{C} \operatorname{Conc}^{*}(\mathcal{A})$ is proper. It is interesting to note, however, that $r(\mathcal{A})$-reflexions do belong to $\mathcal{C C}$ onc $^{*}(\operatorname{lr}(\mathcal{A}))$, so that $\mathcal{S G}$ ph has $(\mathcal{S C o n c}(\operatorname{lr}(\mathcal{A})), \mathcal{D} i s s(\operatorname{lr}(\mathcal{A})))$-factoriza- 
tions, with $\mathcal{S C o n c}(\operatorname{lr}(\mathcal{A}))=\mathcal{C}_{\text {onc }} *(\operatorname{lr}(\mathcal{A}))=\mathcal{H C} \operatorname{Conc}^{*}(\operatorname{lr}(\mathcal{A}))=\mathcal{H C} \operatorname{Conc}^{*}(\mathcal{A})$. This shows in particular properness of the inclusion $\mathcal{C o n c}^{*}(\mathcal{A}) \subset \mathcal{H} \mathcal{C o n c}^{*}(\mathcal{A})$.

REMARKS 4.10.

(1) For $\mathcal{A}$ with $r(\mathcal{A})$ reflective, one easily shows $\mathcal{H C}$ onc $(\mathcal{A})=\{f \mid R f$ iso $\}$, where $R$ denotes the reflector.

(2) Without any further conditions, every morphism in $\mathcal{X}$ can be factored into an $\mathcal{H C}$ onc $^{*}(\mathcal{A})$-morphism followed by a $\mathcal{D}$ iss $(\mathcal{A})$-morphism

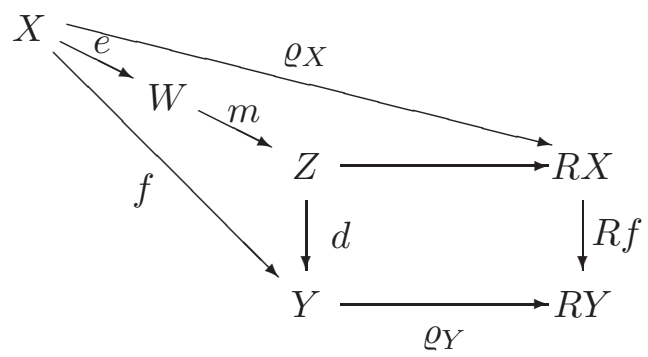

Since $R f \in \mathcal{D} \operatorname{iss}(\mathcal{A})$ (cf. 4.6(1)), also its pullback $d$ along $\varrho_{Y}$ lies in $\operatorname{Diss}(\mathcal{A})$. Let $w=m \cdot e$ be the (regular epi, mono)-factorization of the induced morphism $w: X \rightarrow Z$. Then $f=(d \cdot m) \cdot e$, with $e \in \mathcal{H C o n c} c^{*}(\mathcal{A})$ and $d \cdot m \in \operatorname{Diss}(\mathcal{A})$ (cf. 4.2).

However, since $\operatorname{Diss}(\mathcal{A})^{\perp}=\operatorname{Conc}^{*}(\mathcal{A})$ may be properly contained in $\mathcal{H C}_{\text {onc }}^{*}(\mathcal{A})$, in general $\left(\mathcal{H C}\right.$ onc $^{*}(\mathcal{A}), \mathcal{D}$ iss $\left.(\mathcal{A})\right)$ is not an orthogonal factorization system.

(3) As outlined in [7], an $\left(\mathcal{H C O n c}(\mathcal{A}), \mathcal{H C}\right.$ onc $\left.(\mathcal{A})_{\perp}\right)$-factorization of $f$ may be constructed by taking (in the terminology of (2)) for $m$ the intersection of all those strong subobjects of $Z$ which lie in $(\mathcal{H C} \text { onc }(\mathcal{A}))^{\perp}$ and through which $w$ factors, and for $e$ the induced morphism. Then again $f=(d \cdot m) \cdot e$ is the desired factorization. But note specifically that the first factor of this factorization system is not $\mathcal{H C}$ onc $^{*}(\mathcal{A})$ but $\mathcal{H C}$ onc $(\mathcal{A})$.

(4) $\left(\mathcal{H C} \operatorname{conc}^{*}(\mathcal{A}),\left(\mathcal{H C} \operatorname{conc}^{*}(\mathcal{A})\right)_{\perp}\right)$-factorizations can be constructed à la Herrlich-Salicrup-Vázquez [21], as follows: for $f: X \rightarrow Y$, let $m \cdot e=\left\langle f, \varrho_{X}\right\rangle$ : $X \rightarrow Y \times R X$ be a (regular epi, mono)-factorization, and $f=(d \cdot m) \cdot e$ with $d$ the projection constitutes a left $\mathcal{H C} \operatorname{Conc}^{*}(\mathcal{A})$-factorization. This gives an orthogonal factorization system since $\mathcal{H C o n c}{ }^{*}(\mathcal{A})=\{f \mid R f$ iso $\} \cap \operatorname{Epi}^{*}(\mathcal{X})$ is closed under composition. But in general $\left(\mathcal{H C} \operatorname{Conc}^{*}(\mathcal{A})\right)_{\perp} \neq \operatorname{Diss}(\mathcal{A})$ since otherwise $\mathcal{H C}$ onc $^{*}(\mathcal{A})=\mathcal{C}$ onc $^{*}(\mathcal{A})$.

\section{Separated versus Dissonant, Connected versus Concordant}

PROPOSITION 5.1. For a closure operator $c$ w.r.t. $(\mathcal{E}, \mathcal{M})$, consider the conditions

(i) $c$ is hereditary, 
(ii) every morphism $p: A \rightarrow X$ with $A \in \nabla(c)$ is c-connected,

(iii) every regular epimorphism $p: A \rightarrow X$ with $A \in \nabla(c)$ is c-connected.

Then (i) $\Rightarrow$ (ii) $\Rightarrow$ (iii), and $\mathcal{C o n c}^{*}(\nabla(c)) \subseteq \mathcal{C O n n}^{*}(c) \Rightarrow$ (iii) $\Rightarrow \mathcal{S} e p(c) \subseteq$ $\operatorname{Diss}(\nabla(c))$.

Proof. (i) $\Rightarrow$ (ii) With 2.3(1), c-connectedness of $p$ follows from $c$-connectedness of $(A \rightarrow X \rightarrow 1)$. (ii) $\Rightarrow$ (iii) is trivial. That (iii) is a necessary condition for the inclusion $\mathcal{C}_{o n c} c^{*}(\nabla(c)) \subseteq \mathcal{C}$ onn ${ }^{*}(c)$ follows from the immediate fact that a regular epimorphism $p: A \rightarrow X$ with $A \in \mathcal{A}=\nabla(c)$ is $\mathcal{A}$-concordant. (Note that because of pullback stability of $\mathcal{E}, \mathcal{A}$ is in fact closed under $\mathcal{E}$-images; cf. [9], 4.1.)

Sufficiency of (iii) for the inclusion $\operatorname{Sep}(c) \subseteq \mathcal{D}$ iss $(\nabla(c))$ is shown, as follows. For $f: X \rightarrow Y c$-separated, consider $h: A \rightarrow X$ with $A \in \nabla(c)$; without loss of generality, we may assume $h \in \mathcal{M}$. In the (regular epi, mono)-factorization $f \cdot h=n \cdot p$, the regular epimorphism $p$ is $c$-connected, by hypothesis. Since $\mathcal{S} e p(c) \subseteq \mathcal{C}$ onn $(c)_{\perp}$ one obtains a morphism $t$ with $t \cdot p=h$ and $f \cdot t=n$. The first equation forces $p$ to be monic, hence an isomorphism, so that the second equation makes $f \cdot h \cong n$ monic. Consequently, $f$ is $\nabla(c)$-dissonant.

5.2. In what follows we wish to derive sufficient conditions for the inclusion $\mathcal{C}_{\text {onn }}^{*}(\nabla(c)) \subseteq \mathcal{C}_{\text {onn }}^{*}(c)$. But first we observe with 3.2 and 4.3 that this inclusion necessarily implies $\mathcal{S C o n c}(\nabla(c)) \subseteq \mathcal{W C}$ onn $(c)$. Furthermore:

PROPOSITION. If $\mathcal{X}$ has generalized coequalizers, condition (ii) of 5.1 implies

$$
\mathcal{S C o n c}(\nabla(c)) \subseteq \mathcal{W C o n n}(c) \text {. }
$$

Proof. Let us compare the factorizations $h_{f} \cdot q_{f}=f=d_{f} \cdot p_{f}: X \rightarrow Y$ of 3.2 and 4.3. By hypothesis, for every $a: A \rightarrow X$ with $A \in \nabla(c)$, the morphism $f \cdot a$ is $c$-connected, hence $\delta_{f \cdot a}$ is $c$-dense. Hence there is, for every $a$, a morphism $t_{a}$ rendering the diagram

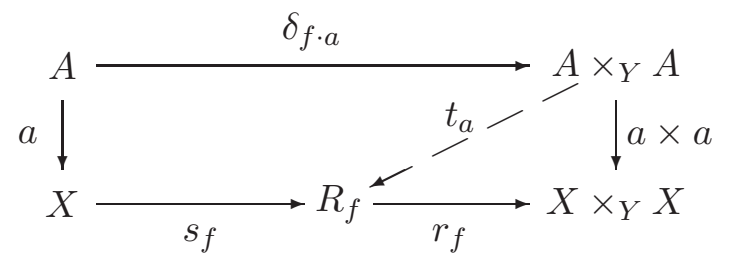

commutative. But then there must be a morphism $t: P_{f} \rightarrow Q_{f}$ with $t \cdot p_{f}=q_{f}$ and $h_{f} \cdot t=d_{f}$, and $t$ must be epic. Consequently, if $d_{f}$ is an isomorphism, so is $h_{f}$.

5.3. Recall that both $\Delta(c)$ and $r(\nabla(c))$ are closed under mono-sources in $\mathcal{X}$, hence reflective if $\mathcal{X}$ has products and is $\mathcal{E}$-cowellpowered. Trivially one has the inclusion $\Delta(c) \subseteq r(\nabla(c))$. Furthermore: 
PROPOSITION. Let c be weakly hereditary and assume $\mathcal{D} i s s(\nabla(c)) \subseteq \mathcal{S} e p(c)$. Then $\Delta(c)=r(\nabla(c))$ if $\Delta(c)$ is reflective in $\mathcal{X}$.

Proof. The $\Delta(c)$-reflexion $e: X \rightarrow X^{\prime}$ of $X \in r(\nabla(c))$ is $r(\nabla(c))$-dissonant by 4.6(1), hence $c$-separated by hypothesis. With $2.2(2)$, the composite $(X \rightarrow$ $\left.X^{\prime} \rightarrow 1\right)$ is $c$-separated, hence $X \in \Delta(c)$.

THEOREM 5.4. Let $c$ be hereditary and assume that $\Delta(c)=r(\nabla(c))$ is reflective in $\mathcal{X}$. Then $\mathcal{C o n c}^{*}(\nabla(c)) \subseteq \mathcal{C O n n}^{*}(c)$ if and only if $\mathcal{S e p}(c) \subseteq \mathcal{D}$ iss $(\nabla(c))$ and $\Delta(c)$-reflexions are c-connected.

Proof. The 'only if' part follows from 5.1 and 4.6(2). Conversely, $\mathcal{S e p}(c) \subseteq$ $\operatorname{Diss}(\nabla(c))$ implies with $4.2 \operatorname{Conc}^{*}(\nabla(c)) \subseteq(\mathcal{S} e p(c))^{\perp}$, which gives $\operatorname{Conc}^{*}(\nabla(c)) \subseteq \mathcal{C}_{\text {onn }}^{*}(c)$ with 2.4 under the given hypotheses.

5.5. Recall that, for a full subcategory $\mathcal{B}$ of $\mathcal{X}$, the $\mathcal{B}$-regular closure of $m \in$ sub $X$ is defined by

$$
\operatorname{reg}_{X}^{\mathcal{B}}(m)=\bigwedge\{\text { equalizer }(u, v) \mid u, v: X \rightarrow B, B \in \mathcal{B}, u \cdot m=v \cdot m\},
$$

where the multiple pullback defining the meet is assumed to exist in $\mathcal{X}$.

THEOREM. Let the full subcategory $\mathcal{A}$ of $\mathcal{X}$ be closed under $\mathcal{E}$-images and generating (cf. 4.5), and assume $r(\mathcal{A})$ to be reflective with all reflexions in $\mathcal{C C}$ onc $(\mathcal{A})$. Then

$$
\mathcal{D i s s}(\mathcal{A}) \subseteq \mathcal{S} e p\left(\operatorname{reg}^{r(\mathcal{A})}\right) \text { and } \mathcal{C o n n}^{*}\left(\operatorname{reg}^{r(\mathcal{A})}\right) \subseteq \mathcal{C o n c}^{*}(\mathcal{A}) .
$$

Proof. Let $f: X \rightarrow Y$ be $\mathcal{A}$-dissonant, and let $e: X \rightarrow X^{\prime}$ be the $r(\mathcal{A})$ reflexion of $X$. We form the equalizer $m: M \rightarrow X \times_{Y} X$ of $\left(e \cdot f_{1}, e \cdot f_{2}\right)$ and obtain a morphism $t: X \rightarrow M$ with $m \cdot t=\delta_{f}$. For every $a: A \rightarrow M$ with $A \in \mathcal{A}$, let $u=a^{\prime} \cdot e^{\prime}$ be an $(\mathcal{E}, \mathcal{M})$-factorization of $u=e \cdot f_{1} \cdot m \cdot a$. For $i=1,2$, there are morphisms $t_{i}$ rendering the diagram

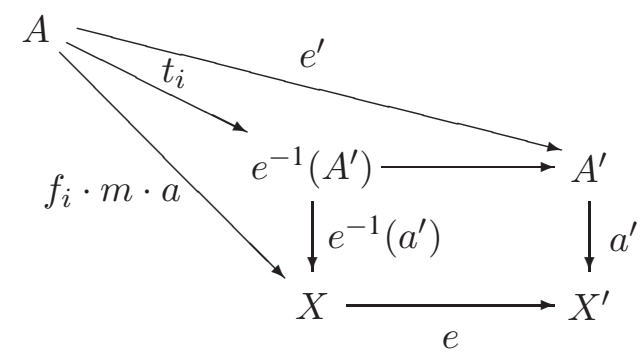

commutative. By hypothesis on $\mathcal{A}$ and $e$, one has $A^{\prime} \in \mathcal{A}$, so that $e^{-1}\left(a^{\prime}\right)$ factors as $e^{-1}\left(a^{\prime}\right)=\tilde{a} \cdot s$ with the codomain of $\tilde{a}$ belonging to $\mathcal{A}$. Consequently, $f \cdot \tilde{a}$ is monic, and

$$
f \cdot \tilde{a} \cdot s \cdot t_{1}=f \cdot f_{1} \cdot m \cdot a=f \cdot f_{2} \cdot m \cdot a=f \cdot \tilde{a} \cdot s \cdot t_{2}
$$


gives $s \cdot t_{1}=s \cdot t_{2}$. Hence, $f_{1} \cdot m \cdot a=f_{2} \cdot m \cdot a$ for all $a$, and therefore $f_{1} \cdot m=f_{2} \cdot m$ since $\mathcal{A}$ is generating. But now $m$ must factor through the equalizer $\delta_{f}$ of $\left(f_{1}, f_{2}\right)$, so that $t$ must be an isomorphism. Therefore $\delta_{f}$ is the equalizer of $\left(e \cdot f_{1}, e \cdot f_{2}\right)$. Since the codomain $X^{\prime}$ of $e$ belongs to $r(\mathcal{A})$, this shows that $f$ is $\operatorname{reg}^{r(\mathcal{A})}$-separated.

The second inclusion follows formally with 4.2 and 2.4:

$$
\mathcal{C o n n}^{*}\left(\operatorname{reg}^{r(\mathcal{A})}\right) \subseteq \mathcal{S} e p\left(\operatorname{reg}^{r(\mathcal{A})}\right)^{\perp} \subseteq \mathcal{D} i s s(\mathcal{A})^{\perp}=\mathcal{C}_{\operatorname{conc}}^{*}(\mathcal{A}) .
$$

5.6. Combining 5.1-5.5 in the case $\mathcal{A}=\nabla(c)$ we obtain:

COROLLARY. Let $c$ be hereditary, with $\nabla(c)$ generating and $\Delta(c)$ reflective in $\mathcal{X}$ such that all reflexions belong to $\mathcal{C}$ onn $(c) \cap \mathcal{C C}$ onc $(\nabla(c))$. Then

$$
\mathcal{S e p}(c)=\operatorname{Diss}(\nabla(c)) \text { and } \operatorname{Conn}^{*}(c)=\operatorname{Conc}^{*}(\nabla(c))
$$

if and only if $\Delta(c)=r(\nabla(c))$.

Proof. Since $c \leqslant \operatorname{reg}^{\Delta(c)}$ for every closure operator $c$, in case $\Delta(c)=r(\nabla(c))$ one has the inclusions

$$
\begin{aligned}
& \mathcal{S e p}(c) \subseteq \mathcal{D} i s s(\nabla(c)) \subseteq \mathcal{S} e p\left(\operatorname{reg}^{r(\nabla(c))}\right)=\mathcal{S} e p\left(\operatorname{reg}^{\Delta(c)}\right) \subseteq \mathcal{S} e p(c), \\
& \mathcal{C o n c}^{*}(\nabla(c)) \subseteq \mathcal{C} \operatorname{onn}^{*}(c) \subseteq \mathcal{C o n n}^{*}\left(\operatorname{reg}^{\Delta(c)}\right) \\
& \quad=\mathcal{C o n n}^{*}\left(\operatorname{reg}^{r(\nabla(c))}\right) \subseteq \mathcal{C}_{\text {onc }}(\nabla(c)) .
\end{aligned}
$$

Necessity of the condition $\Delta(c)=r(\nabla(c))$ was shown in 5.3.

EXAMPLE 5.7. For the $b$-closure as in 3.7(1), $\nabla(b)$ is the subcategory of indiscrete spaces, which is also the right-constant subcategory of $\Delta(b)$. Since $T_{0^{-}}$ reflexions satisfy the condition of 5.6, this corollary therefore gives coincidence of $\nabla(b)$-concordant quotient maps and $\nabla(b)$-dissonant maps with $b$-connected quotient maps and $b$-separated maps, respectively.

The conditions in the Corollary are quite restrictive. Further to Theorem 5.5, the question remains whether the stated inclusions may be proper and, more generally, whether $\operatorname{Diss}(\mathcal{A})$ is of the form $\mathcal{S e p}(c)$ for any closure operator $c$; similarly for $\operatorname{Conc}^{*}(\mathcal{A})$. The answer follows.

\section{Failure of Closure Operators for Monotone-Light and Concordant-Dissonant}

6.1. For $\mathcal{A}$ the subcategory of connected spaces of $\mathcal{T}$ op, the prefix in $\mathcal{A}$-dissonant and $\mathcal{A}$-concordant is omitted.

PROPOSITION. There is no closure operator c of $\mathcal{T}$ op such that $\mathcal{S} e p(c)$ is exactly the class of dissonant maps. 
Proof. Assume $\operatorname{Diss}(\mathcal{A})=\operatorname{Sep}(c)$ for some closure operator $c$. Since $X \in$ $\Delta(c)$ iff $(X \rightarrow 1) \in \mathcal{S} e p(c)$ and $X \in r(\mathcal{A})$ iff $(X \rightarrow 1) \in \mathcal{D} i s s(\mathcal{A})$, we must have $r(\mathcal{A})=\Delta(c)$, hence $c \leqslant \operatorname{reg}^{r(\mathcal{A})}$. This implies $\mathcal{S e p}\left(\operatorname{reg}^{r(\mathcal{A})}\right) \subseteq \mathcal{S} e p(c)=$ $\mathcal{D} i s s(\mathcal{A})$. Hence, in order to complete the proof, it suffices to exhibit a $\operatorname{reg}^{r(\mathcal{A})}$ separated map which is not dissonant. For this we employ again the map

$$
f: I \rightarrow S^{1}, \quad x \mapsto(\cos 2 \pi x, \sin 2 \pi x)
$$

of 2.6(2), which is not dissonant (since it is not monic although its domain is connected). However, $f$ is $\operatorname{reg}^{r(\mathcal{A})}$-separated since $\delta_{f}: I \rightarrow I \times{ }_{S^{1}} I$ is the equalizer of two maps with hereditarily disconnected codomain $D$; in fact, one may take $D$ to be the 3-point discrete space and define two continuous maps $I \times{ }_{S^{1}} I \rightarrow D$ which leave the diagonal fixed and interchange the two isolated points of $I \times{ }_{S^{1}} I$.

PROPOSITION 6.2. There is no closure operator $c$ of $\mathcal{T}$ op such that $\mathcal{C}_{\text {onn }}{ }^{*}(c)$ is exactly the class of concordant quotient maps.

Proof. Assume $\mathcal{C}_{\text {onc }}{ }^{*}(\mathcal{A})=\mathcal{C}$ onn $^{*}(c)$. With the same argumentation as in 6.1 , this implies $\nabla(c)=\mathcal{A}$. The map $f: I \rightarrow S^{1}$ is concordant, hence $c$ connected, so that $\delta_{f}$ must be $c$-dense. There is a commutative diagram

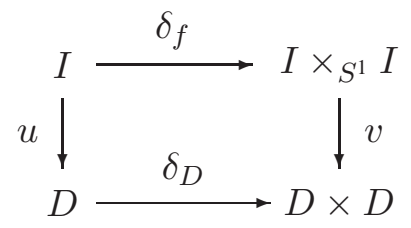

with $D=\{0,1\}$ discrete, $u$ the map constant 0 , and $v$ mapping the two isolated points to $(1,0)$ and $(0,1)$. By $c$-continuity of $v,\{(0,0),(1,0),(0,1)\}=$ $v\left(c\left(\delta_{f}\right)\right) \subseteq c\left(v\left(\delta_{f}\right)\right) \subseteq c\left(\delta_{D}\right)$. Since trivially $(1,1) \in c\left(\delta_{D}\right)$, this implies that $D \in \nabla(c)$ is connected - a contradiction.

PROPOSITION 6.3. There is no closure operator $c$ of $\mathcal{T}$ op such that $\mathcal{C}_{\text {onn }}{ }^{*}(c)$ is exactly the class of monotone quotient maps.

Proof. Assume that $\mathcal{C}_{\text {onn }}^{*}(c)$ is the class of monotone quotient maps for some closure operator $c$. Then $\nabla(c)=\mathcal{A}$ since $X \rightarrow 1$ is monotone iff $X$ is connected; hence $c \geqslant \operatorname{coreg}^{\mathcal{A}}$. The map $g: I \rightarrow I$ of 2.6(2) is coreg ${ }^{\mathcal{A}}$-connected, because it is coreg $\mathcal{A}^{\prime}$-connected, for $\mathcal{A}^{\prime}$ the subcategory of path-connected spaces, and coreg $\mathcal{A}^{\prime} \leqslant \operatorname{coreg}^{\mathcal{A}}$ since $\mathcal{A}^{\prime} \subset \mathcal{A}$. Hence $g$ is $c$-connected but not monotone (in fact, $g$ is light) - a contradiction.

PROPOSITION 6.4. There is no closure operator c of $\mathcal{T}$ op such that $\mathcal{S} e p(c)$ is exactly the class of light maps.

Proof. Assume that there is a closure operator $c$ such that $\operatorname{Sep}(c)$ is the class of light maps. The map $g: I \rightarrow I$ of 2.6(2) is light. Now, for all $x \neq y$ in $I$ there is a continuous map $h_{(x, y)}: I \times I \rightarrow I \times_{I} I$ keeping the diagonal $\delta_{I}$ fixed and 
mapping $(x, y)$ to a point outside the diagonal $\delta_{g}$. Hence $(x, y) \notin c\left(\delta_{I}\right)$ since $h_{(x, y)}\left(c\left(\delta_{I}\right)\right) \leqslant c\left(h\left(\delta_{I}\right)\right)=c\left(\delta_{g}\right)=\delta_{g}$, that is: $\delta_{I}$ is $c$-closed. But this implies that $I$ is $c$-separated so that the map $I \rightarrow 1$ must be light: contradiction.

\section{Failure of the (Co-)Diagonal Theorem in the Absence of Enough Points}

7.1. It was shown in [19] that any strongly epireflective subcategory $\mathcal{B}$ in the category $\mathcal{X}$ in which the terminal object is a generator must be of the form $\Delta(c)$ for some closure operator $c$. This Diagonal Theorem was generalized in [9], where we assumed $\mathcal{B}$ to be closed under monosources, with the latter being detected by quasipoints (so that $\left(m_{i}: X \rightarrow Y_{i}\right)_{i}$ is monic if $m_{i} \cdot x=m_{i} \cdot y$ for all $x, y: T X \rightarrow X$, with $T X$ appearing in the $(\mathcal{E}, \mathcal{M})$-factorization of $X \rightarrow 1)$. Now we show that the condition on $\mathcal{X}$ (having enough points, or at least enough quasipoints) is essential for the Diagonal Theorem to hold:

THEOREM. The category $\mathcal{T}$ op $/ S^{1}$ contains a right constant strongly epireflective full subcategory which is not presentable in the form $\Delta(c)$ for any closure operator $c$ of $\mathcal{T}_{o p} / S^{1}$ w.r.t. its (surjective, embedding)-factorization structure.

Proof. Let $\mathcal{B}$ be the full subcategory of $\mathcal{T} o p / S^{1}$ given by the dissonant maps with codomain $S^{1}$. Since $\mathcal{T} o p$ has (concordant quotient, dissonant)-factorizations (cf. 4.8), $\mathcal{B}$ is strongly epireflective in $\mathcal{T}_{o p} / S^{1}$ (cf. 1.1). If we had $\mathcal{B}=\Delta(c)$ in $\mathcal{T}$ op $/ S^{1}$, necessarily $\mathcal{B}=\Delta\left(\mathrm{reg}^{\mathcal{B}}\right)$, with $\operatorname{reg}^{\mathcal{B}}$ the $\mathcal{B}$-regular closure operator on $\mathcal{T} o p / S^{1}$. But an easy modification of the proof given in 6.1 shows that the map $f: I \rightarrow S^{1}$ belongs to $\Delta\left(\mathrm{reg}^{\mathcal{B}}\right)$, although it is not dissonant. In fact, $\delta_{f}$ is the equalizer of two maps over $S^{1}$ with dissonant codomain $q$, as follows:

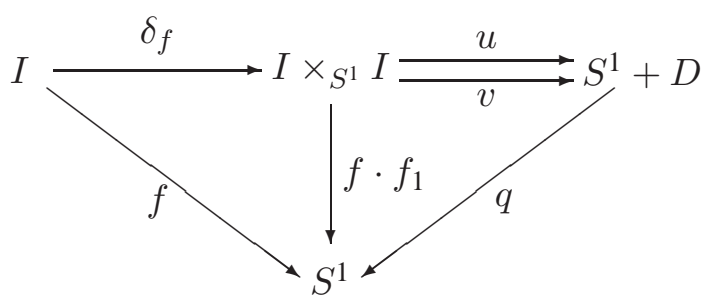

Here $D=\{a, b\}=q^{-1}\{f(0)=f(1)\} \cap D=u\{(0,1),(1,0)\}=v\{(0,1),(1,0)\}$ is discrete.

7.2. In [9], Theorem 3.4, we gave a sufficient condition for a full subcategory $\mathcal{A}$ of the category $\mathcal{X}$ to be of the form $\nabla(c)$, assuming $\mathcal{X}$ to have enough quasipoints. Again, good behaviour of (quasi-)points turns out to be crucial for the Co-Diagonal Theorem to hold.

THEOREM. The category $\mathcal{T}_{\text {op }} / S^{1}$ contains a left constant full subcategory which is not presentable in the form $\nabla(c)$ for any closure operator $c$ of $\mathcal{T}$ op $/ S^{1}$. 
Proof. Let $\mathcal{A}$ be the full subcategory of $\mathcal{T}$ op $/ S^{1}$ given by all concordant maps with codomain $S^{1}$, and assume $\mathcal{A}=\nabla(c)$. Again, we consider the concordant map $f: I \rightarrow S^{1}$ as well as the non-concordant map $g:=f \cdot f_{1}: I \times_{S^{1}} I \rightarrow S^{1}$. Our proof is complete once we have shown that with $f$ also $g$ must belong to $\nabla(c)$.

In fact, since $Y=I \times{ }_{S^{1}} I \cong I+D$ with $D$ a 2-point discrete space, the kernelpair $W=Y \times{ }_{S^{1}} Y$ of $g$ is of the form $Y+E$ with $E$ a 12-point discrete space. One has commutative diagrams over $S^{1}$

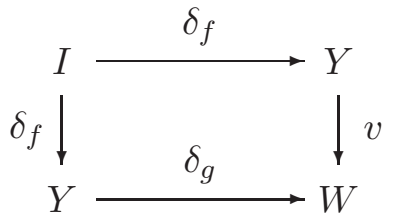

with $v$ mapping $I$ identically and $D$ to $E$. Considering various maps $v$, as in 6.2 one shows $c\left(\delta_{g}\right)=W$, hence $g \in \nabla(c)$.

REMARK 7.3. The terminal object of $\mathcal{T}_{o p} / S^{1}$ fails to be a generator, but its preterminal objects are generating. In fact, the maps $1 \rightarrow S^{1}$ form a generator of Top $/ S^{1}$, which shows that in the (Co-)Diagonal Theorem as formulated in [9], quasipoints may not be traded for prepoints.

\section{Acknowledgement}

The authors acknowledge partial financial assistance by a NATO Collaborative Research Grant (no. 940847), by the Centro de Matemática da Universidade de Coimbra, and by the Natural Sciences and Engineering Research Council of Canada.

\section{References}

1. Arhangel'skiǐ, A. and Wiegandt, R.: Connectednesses and disconnectednesses in topology, Gen. Topol. Appl. 5 (1975), 9-33.

2. Barr, M.: Exact Categories, Lecture Notes in Math. 236, Springer, Berlin, 1971, pp. 1-120.

3. Barr, M.: On categories with effective unions, Categorical Algebra and Its Applications, Lecture Notes in Math. 1348, Springer, Berlin, 1988, pp. 19-35.

4. Börger, R.: Making factorizations compositive, Comm. Math. Univ. Carolina 32 (1991), 749_ 759.

5. Börger, R. and Tholen, W.: Concordant-dissonant and monotone-light, in: Proc. Conf. Categorical Topology, Toledo, Ohio, 1983, Heldermann Verlag, Berlin, 1984, pp. 90-107.

6. Carboni, A., Janelidze, G., Kelly, G. M. and Paré, R.: On localization and stabilization for factorization systems, Applied Categorical Structures 5 (1997), 1-58.

7. Cassidy, C., Hébert, M. and Kelly, G. M.: Reflective subcategories, localizations and factorization systems, J. Austral. Math. Soc., Ser. A 38 (1985), 287-329.

8. Clementino, M. M., Giuli, E. and Tholen, W.: Topology in a category: compactness, Port. Math. 53 (1996), 397-433.

9. Clementino, M. M. and Tholen, W.: Separation versus connectedness, Topology Appl. 75 (1997), 143-181. 
10. Collins, P. J.: Concordant mappings and the concordant-dissonant factorisation of an arbitrary continuous function, Proc. Amer. Math. Soc. 27 (1971), 587-591.

11. Collins, P. J. and Dyckhoff, R.: Connexion properties and factorisation theorems, Quaestiones Math. 2 (1977), 103-112.

12. Dikranjan, D.: Semiregular closure operators and epimorphisms in topological categories, Suppl. Rend. Circ. Mat. Palermo, Serie II 29 (1992), 105-160.

13. Dikranjan, D. and Giuli, E.: Closure operators I, Topology Appl. 27 (1987), 129-143.

14. Dikranjan, D. and Tholen, W.: Categorical Structure of Closure Operators, with Applications to Topology, Algebra and Discrete Mathematics, Kluwer Academic Publishers, 1995.

15. Dyckhoff, R.: Categorical cuts, Topology Appl. 6 (1976), 291-295.

16. Eilenberg, S.: Sur les transformations continues d'espaces metriques compacts, Fund. Math. 22 (1934), 292-296.

17. Freyd, P. J. and Kelly, G. M.: Categories of continuous functors, I, J. Pure Appl. Algebra 2 (1972), 169-191. Erratum ibid. 4 (1974), 121.

18. Giuli, E. and Hušek, M.: A diagonal theorem for epireflective subcategories of $\mathcal{T}_{o p}$ and cowellpoweredness, Ann. Mat. Pura Appl. 145 (1986), 337-346.

19. Giuli, E., Mantovani, S. and Tholen, W.: Objects with closed diagonals, J. Pure Appl. Algebra 51 (1988), 129-140.

20. Herrlich, H.: Topologische Reflexionen und Coreflexionen, Lecture Notes in Math. 78, Springer, Berlin, 1968.

21. Herrlich, H., Salicrup, G. and Vázquez, R.: Dispersed factorization structures, Can. J. Math. 31 (1979), 1059-1071.

22. Herrlich, H., Salicrup, G. and Vázquez, R.: Light factorization structures, Quaestiones Math. 3 (1979), 189-213.

23. James, I. M.: Fibrewise Topology, Cambridge University Press, Cambridge, New York, 1989.

24. Janelidze, G. and Tholen, W.: Functorial factorization, wellpointedness and separability, preprint, 1997.

25. Kelly, G. M.: Monomorphisms, epimorphisms and pull-backs, J. Austral. Math. Soc., Ser. A 9 (1969), 124-142.

26. Kelly, G. M.: A unified treatment of transfinite constructions for free algebras, free monoids, colimits, associate sheaves, and so on, Bull. Austral. Math. Soc. 22 (1980), 1-83.

27. Korostenski, M. and Tholen, W.: Factorization systems as Eilenberg-Moore algebras, J. Pure Appl. Algebra 85 (1993), 57-72.

28. Linton, F. E. J.: Coequalizers in categories of algebras, Lecture Notes in Math. 80, Springer, Berlin, 1969, pp. 75-90.

29. MacDonald, J. and Tholen, W.: Decomposition of morphisms into infinitely many factors, Lecture Notes in Math. 962, Springer, Berlin 1982, pp. 175-182.

30. Michael, E.: Cuts, Acta Math. 111 (1964), 1-36.

31. Preuß, G.: Eine Galoiskorrespondenz in der Topologie, Monatsh. Math. 75 (1971), 447-452.

32. Pumplün, D.: Universelle und spezielle Probleme, Math. Ann. 198 (1972), 131-146.

33. Ringel, C. M.: Diagonalisierungspaare I, Math. Z. 112 (1970), 248-266.

34. Strecker, G. E.: Component properties and factorizations, Math. Center Tracts 52 (1974), 123-140.

35. Tholen, W.: Factorizations, localizations and the orthogonal subcategory problem, Math. Nachr. 114 (1983), 63-85.

36. Tholen, W.: Prereflections and reflections, Comm. in Algebra 14 (1987), 717-740.

37. Tholen, W.: Semi-topological functors I, J. Pure Appl. Algebra 15 (1979), 53-73.

38. Tholen, W.: Objects with closed diagonals, in: Proc. Workshop on Categorical Topology (L'Aquila, Italy 1994), Kluwer Academic Publishers, 1995.

39. Tiller, J. A.: Component subcategories, Quaestiones Math. 4 (1980), 19-40.

40. Whyburn, G. T.: Non-alternating transformations, Amer. J. Math. 56 (1934), 294-302.

41. Wyler, O. and Ehrbar, H.: Images in categories as reflections, Cahiers Topologie Géom. Différentielle Catégoriques 28 (1987), 143-159. 